\title{
Holomorphicity and modularity in Seiberg-Witten theories with matter
}

\author{
Min-xin Huang ${ }^{a}$ and Albrecht Klemm ${ }^{b}$ \\ a Theory Division, Department of Physics, CERN, \\ CH-1211 Geneva, Switzerland \\ ${ }^{b}$ Bethe Center for Theoretical Physics and Physikalisches Institut, Universität Bonn, \\ Nussallee 12, D-53115 Bonn, Germany \\ E-mail: minxin.huang@cern.ch, aklemm@th.physik.uni-bonn.de
}

ABSTRACT: We calculate the gravitational corrections to the effective action of $\mathrm{N}=2 \mathrm{SU}(2)$ Seiberg-Witten theory with matter using modularity, the holomorphic anomaly equation and expected behavior at the boundaries of the moduli space. As in pure gauge theory we show that the gap condition at the dyon singularities completely fixes the gravitational corrections. We discuss the behavior of the gravitational corrections at the conformal points. We compare our results with the recursive solution of the loop equation in the matrix model approach, which provides in addition open amplitudes.

KEYwords: Supersymmetric gauge theory, Matrix Models, Supersymmetry and Duality

ARXiv EPRINT: 0902.1325 


\section{Contents}

1 Introduction $\quad 2$

2 SU(2) Seiberg-Witten theory with massless hypermultiplets 4

2.1 Topological string amplitude $F^{(g)}$ as polynomials of $\hat{E}_{2} \quad 6$

$\begin{array}{ll}2.2 N_{f}=1 & 11\end{array}$

$\begin{array}{ll}2.3 N_{f}=2 & 13\end{array}$

$\begin{array}{lll}2.4 N_{f}=3 & 15\end{array}$

3 SU(2) Seiberg-Witten theory with massive hypermultiplets 17

$\begin{array}{ll}3.1 & \text { The prepotential } F^{(0)} \\ 3.2 & 17\end{array}$

$\begin{array}{lll}3.2 & \text { Gravitational couplings } & 19\end{array}$

3.3 Fixing the holomorphic ambiguity with dual expansions 20

3.4 Comments on the $N_{f}=2,3$ cases and integrability 21

4 SU(2) Seiberg-Witten theory at superconformal points 21

$4.1 N_{f}=1, m_{1}=\frac{3 \Lambda_{1}}{4} \quad 22$

$4.2 N_{f}=2, m_{1}=m_{2}= \pm \frac{\Lambda_{2}}{2} \quad 24$

$4.3 N_{f}=3, m_{1}=m_{2}=m_{3}=\frac{\Lambda_{3}}{8} \quad 25$

5 The non-compact limit of Calabi-Yau compactifications 26

$\begin{array}{ll}5.1 & \text { Special geometry and rigid special geometry } 27\end{array}$

$\begin{array}{lll}5.2 & \text { The non-compact limit } 30\end{array}$

$\begin{array}{ll}5.3 \text { Monodromy action } & 31\end{array}$

6 Matrix model approach 33

6.1 Review of the formalism 34

$\begin{array}{lll}\text { 6.2 Calculations of open and close amplitudes } & 38\end{array}$

6.3 Holomorphic anomaly equation for open amplitudes 39

6.4 Boundary condition for open topological amplitudes 42

$\begin{array}{lll}7 & \text { Future directions } & 43\end{array}$

$\begin{array}{ll}\text { A Nekrasov's calculations } & 44\end{array}$ 


\section{Introduction}

It was demonstrated in [1] for pure $N=2$ supersymmetric $\mathrm{SU}(2)$ gauge theory that the modular symmetry, the holomorphic anomaly equation and the gap condition at the singularities, where dyons become massless, fix all gravitational corrections. Here we will extend this approach to asymptotically free $N=2$ gauge theories with matter. The method we provide extends to several integrable systems which are connected to $N=2$ gauge theories. Let us first review these interrelations.

When the gauge theory is coupled to gravity, the coupling of the selfdual curvature tensor $R_{+}$to the graviphoton field strength $F_{+}$, i.e. $F^{(g)}(\underline{a}) R_{+}^{2} F_{+}^{2 g-2}$, depends on the moduli fields $\underline{a}$ and this dependence is exactly calculable within the topological sector of the theory. An important point is that in different regions of the moduli there are different canonically conjugated coordinates and the different expansion of the $F^{(g)}$ are not just analytic continuations of each other. They are rather related by the wave function transformation of $Z=\exp \left(\sum_{g=0}^{\infty} \lambda^{2 g-2} F_{g}(\underline{a})\right)$ [2], which is closely related to the modular and (an)holomorphic properties of the $F^{(g)}(\underline{a})[1,3]$. e.g. in the weak coupling regime the $F^{(g)}(\underline{a})$ can be calculated from the field theory point of view using localization in the spacetime instanton moduli space $[4,5]$, but to obtain from this expansion the dual expansions e.g. at the monopole points or the conformal points, also known as Argyres-Douglas points, one has to use the wave function transformation [1-3].

$N=2$ gauge theories are related to Type II string theory on non-compact Calabi-Yau manifolds by geometric engineering [6]. It has been checked that the holomorphic gauge coupling functions and the BPS masses of the gauge theory $[7,8]$ can also be obtained from the topological sector of the Type II string theory on this backgrounds in a double scaling limit which decouples $\alpha^{\prime}$ - and Planck scale effects [6, 9]. In particular [6] discusses the geometric engineering of non-compact toric Calabi-Yau spaces for $\mathrm{SU}(N)$ gauge theories with few fundamental matter fields. In the large radius region of the topological string theory the $F^{(g)}(\underline{t})$ are higher genus world-sheet amplitudes, which depend on the Kähler moduli $\underline{t}$. The field theory $F^{(g)}(a)$ can be obtained in the limit mentioned above [11]. A third approach is to calculate the $F^{(g)}(a)$ in the matrix model formalism suggested by $[12,13]$ in an $\frac{1}{N}$ expansion $[11,14]$.

The common mathematical structure of $N=2$ Seiberg-Witten theories, topological string on non-compact Calabi-Yau spaces and matrix models is a family of Riemann surface $\mathcal{C}$ equipped with a meromorphic differential $\lambda$, which has in general non-vanishing residua.

In the Seiberg-Witten approach to supersymmetric gauge theories $\mathcal{C}$ is the SeibergWitten curve and $\lambda$ the Seiberg-Witten differential $[7,8] .(\mathcal{C}, \lambda)$ can be re-derived from the space-time instanton calculus as was demonstrated in [5]. ${ }^{1}$ In topological string theory on non-compact Calabi-Yau spaces the data $(\mathcal{C}, \lambda)$ arise via mirror symmetry in the Bmodel geometry [6, 17]. In the matrix model approach $\mathcal{C}$ is the spectral curve and $\lambda$ the differential defining the filling fractions and the one point function $[5,12,13,16,18]$. The calculations of $[4,5]$ using instanton calculus, of $[1,19]$ within the topological B-model, and

\footnotetext{
${ }^{1}$ Using [15] one can construct the space-time instanton sums of [5] directly as a matrix model [16].
} 
of $[18,20]$ utilizing the matrix model recursions suggest that the higher genus information can be completely reconstructed from $(\mathcal{C}, \lambda)$.

The $B$ model approach is particularly efficient. It uses for the reconstruction the recursion relation in the genus, known as holomorphic anomaly equation [21]. The latter has a kernel, the holomorphic ambiguity, which is strongly constrained by the symmetry group of theory, but certain discrete data have to be fixed by additional arguments. For pure gauge theory the gap condition at the conifold fixes these discrete data completely [1].

In this work we consider the asymptotically free $\mathrm{SU}(2)$ with $N_{f}=1,2,3$ hypermultiplets in the fundamental representation. Similarly as the topological string theory on the canonical line bundle over a del Pezzo surface with more than one Kähler parameter [19], gauge theories with matter have more parameters than the complex moduli space of the Riemann surface. In gauge theory these parameters are simply the masses of the hypermultiplets. While they make the form of the holomorphic ambiguity more complicated, the requirement that the gap exists for all values of the additional parameter imposes stronger conditions. We find that the latter over constrain the system and that an unique solution exists. The gravitational corrections for gauge theories with matter are therefore completely integrable.

One advantage of the method [1] is that it provides the $F^{(g)}$ in all regions in the moduli space and not just in the asymptotically free region. Argyres, Plesser, Seiberg and Witten found particularly interesting points in the moduli space of SU(2) gauge theory with fundamental matter, where different conformal theories arise [22]. Using the formalism [1], one can analyze the full topological sector of these conformal theories. The coordinate choice and the structure of the topological gauge theory amplitudes near conformal points is very similar as for topological string theory near orbifold points [23, 24].

Let us comment finally on the $N_{f}=4$ case. An geometric engineering limit starting from the Enriques Calabi-Yau manifold has been described in [26]. It was shown there, that the calculation of the $\mathcal{F}_{g}$ on the Enriques Calabi-Yau are governed by the holomorphic anomaly equations of [21]. Also the expression obtained for the $\mathcal{F}_{g}$ in the field theory limit can be written as quasimodular forms after finding ${ }^{2}$ the correct relation between $\tau_{I R}$ and $\tau_{U V}$ [26]. However in these quasimodular expressions we have $\mathcal{F}_{g} \sim E_{2}^{g-1}$ in leading order in $E_{2}$. This implies that the $\mathcal{F}_{g}$ for $N_{f}=4$ does fulfill the holomorphic anomaly equation (2.26) for the non-conformal cases, which implies $\mathcal{F}_{g} \sim E_{2}^{3(g-1)}$.

The paper is organized as follows: In section 2, we first consider the simple case of theory with massless matter. Here the Coulomb modulus $u$ is related to the modular parameter $\tau$ by $J(\tau)=R_{N_{f}}(u)$, where $R_{N_{f}}(u)$ is a rational function and $J$ is the modular invariant $J$-function. The Coulomb moduli space is then a ramified finite multicover of the quotient of the upper half plane $\operatorname{Im}(\tau)>0$ by $\operatorname{PSL}(2, \mathbb{Z})$ and $u(\tau)$ has very simple transformation properties w.r.t. $\operatorname{PSL}(2, \mathbb{Z})$. We review the direct integration, which is an efficient way to solve the holomorphic anomaly equation and write the $F^{(g)}$ as polynomials of modular functions. In section 3 we generalize the analysis to the massive $\mathrm{SU}(2)$ Seiberg-Witten theory. We provide an algorithm that is valid for the case of fundamental

\footnotetext{
${ }^{2}$ In [54] $\tau_{I R}$ and $\tau_{U V}$ were erroneously identified, compare [27].
} 
matter with arbitrary mass, and study the $N_{f}=1$ case in details. This procedure can be straightforwardly generalized to $N_{f}=2,3$. In section 4 we discuss some cases of special fundamental hypermultiplet masses where two mutually non-local singular points in the $u$ plane collide and a non-trivial superconformal field theory appears. Section 5 discusses the emergence of the gauge theory in the non-compact limit of a Calabi-Yau compactification. This provides a connection to the direct integration formalism developed in [25, 26, 28, 29], which is useful to solve the gravitational couplings of higher rank gauge theories entirely from the solutions of the Picard-Fuchs equation. In section 6 we turn to the matrix model approach. We find it particularly complicated to solve the $N_{f}=2$ theory by the approach of [20], point out some restrictions in the application of the open holomophic anomaly of [49] and discuss analytic properties of the open amplitudes.

\section{$2 \mathrm{SU}(2)$ Seiberg-Witten theory with massless hypermultiplets}

The Seiberg-Witten curves ${ }^{3}[7,8] \mathcal{C}_{1}$ for $\mathcal{N}=2$ supersymmetric $\mathrm{SU}(2)$ gauge theory with $N_{f}<4$ flavors are families of elliptic curves given by ${ }^{4}$

$$
y^{2}=C(x)^{2}-G(x)
$$

where $C(x)$ and $G(x)$ are defined as

$$
\begin{array}{llrl}
N_{f}=0: C(x)=x^{2}-u, & G(x) & =\Lambda^{4}, \\
N_{f}=1: C(x)=x^{2}-u, & G(x) & =\Lambda^{3}\left(x+m_{1}\right), \\
N_{f}=2: C(x)=x^{2}-u+\frac{\Lambda^{2}}{8}, & G(x) & =\Lambda^{2}\left(x+m_{1}\right)\left(x+m_{2}\right), \\
N_{f}=3: C(x)=x^{2}-u+\frac{\Lambda}{4}\left(x+\frac{m_{1}+m_{2}+m_{3}}{2}\right) & & G(x)=\Lambda\left(x+m_{1}\right)\left(x+m_{2}\right)\left(x+m_{3}\right) .
\end{array}
$$

Here $u$ is the modulus parameterizing the Coulomb branch and $m_{i}$ are the masses of the hypermultiplets. The genus one curves $\mathcal{C}_{1}$ have two periods $a_{D}$ and $a$ of the meromorphic Seiberg-Witten differential $\lambda$ over the $b$ and the $a$ cycle in $H^{1}\left(\mathcal{C}_{1}, \mathbb{Z}\right)$ respectively. The meromorphic one form $\lambda$ can be written with ${ }^{\prime}=\frac{d}{d x}$ as

$$
\lambda=\frac{\sqrt{2}}{4 \pi i} \frac{x d x}{y}\left[\frac{C(x) G^{\prime}(x)}{2 G(x)}-C^{\prime}(x)\right] .
$$

Physically the periods are the vev's of the scalar component of the $\mathcal{N}=2$ vector multiplet containing the photon and its dual in the infrared respectively.

\footnotetext{
${ }^{3}$ For the $N_{f}=0$ we use the family of curves of [30] rather than the one of [7]. This distinction plays a role in establishing the matrix model connection in section 6 .

${ }^{4}$ One can do a change of variable and write the Seiberg-Witten curve in all cases as

$$
y^{2}=\left(x^{2}-u\right)^{2}-\Lambda^{2 N_{c}-N_{f}} \prod_{i=1}^{N_{f}}\left(x+m_{i}\right)
$$

[31]. Here we use the original notations in [8].
} 
We are interested in calculating the instanton expansion of the prepotential $F^{(0)}$ and its higher genus generalization $F^{(g)}$ all over the moduli space. In the asymptotically free region the prepotential $F^{(0)}(a)$ for the electric $\mathrm{U}(1)$ theory, which determines the exact gauge coupling of the $N=2$ Super-Yang-Mills theory, is related to the periods $a_{D}$ and $a$ by rigid special geometry

$$
\frac{\partial F^{(0)}}{\partial a}=a_{D}
$$

As pointed out in [7] in the dual magnetic region where $a_{D}$ is small, the theory is more suitably described by a magnetic $\mathrm{U}(1)$, whose prepotential is given by $\frac{\partial F_{D}^{(0)}}{\partial a_{D}}=a$.

The higher genus terms $F^{(g)}(a)$ describe the exact moduli dependence of the gravitational corrections

$$
F^{(g)} F_{+}^{2 g-2} R_{+}^{2} .
$$

in the effective Lagrangian, which encodes the coupling of the gauge theory to $N=2$ supergravity. Here $F_{+}$and $R_{+}$are the self-dual part of the graviphoton field strength and of the Ricci curvature respectively. In the weak coupling region $u \rightarrow \infty$ we can compare $F^{(g)}(a)$ to the localization calculations in [4]. The relation of the dual $F_{D}^{(g)}\left(a_{D}\right)$ to the $F^{(g)}(a)$, follows from the quantum mechanical wave function transformation of $Z=$ $\exp \left(\sum_{g=0}^{\infty} \lambda^{2 g-2} F^{(g)}\right)$ and was studied in this context in [3].

In this section, we first consider the simple case where all the hypermultiplets are massless. In this case $u(\tau)$ is a modular invariant function under the projective action on the gauge coupling $\tau \propto \frac{\partial^{2}}{\partial^{2} \tau} F^{(0)}$ of the group $\Gamma_{N_{f}} \in \operatorname{PSL}(2, \mathbb{Z})[32]$. To find the rational function $R_{N_{f}}(u)$ discussed in section 1 one simply brings the curve (2.2) into Weierstrass form

$$
y^{2}=4 x^{3}-g_{2}(u) x-g_{3}(u) .
$$

The rational function is then determined by

$$
J(\tau)=\frac{E_{4}(\tau)^{3}}{E_{4}^{3}(\tau)-E_{6}(\tau)^{2}}=\frac{g_{2}(u)^{3}}{g_{2}(u)^{3}-27 g_{3}(u)^{2}}=: R_{N_{f}}(u) .
$$

Here

$$
\Delta=g_{2}(u)^{3}-27 g_{3}(u)^{2}
$$

is the discriminant of the curve. The group $\Gamma_{N_{f}}$ is the quotient of $\operatorname{PSL}(2, \mathbb{Z})$ by the group interchanging the roots of $u(J)$ and has been determined in [32]. In the mathematical literature the invariant $u(\tau)$ is sometimes called the 'Hauptmodul' of $\Gamma_{N_{f}}$.

The Picard-Fuchs differential equations are fulfilled by all periods over the cycles of $\mathcal{C}_{1}$. In the massless case $\lambda$ has no non-vanishing residua and the two periods $a_{D}$ and $a$ fulfill a second order Picard Fuchs differential equations, ${ }^{5}$ which were derived for $N_{f}=1,2,3$ in [33]

$$
p(u) \frac{d^{2} \Pi}{d u^{2}}+\Pi=0,
$$

\footnotetext{
${ }^{5}$ In the massive case discussed in section 3 there is also a cycle encircling the pole of $\lambda$ picking up the residuum and the differential equations are third order.
} 
where

$$
\begin{aligned}
& N_{f}=1: p(u)=4 u^{2}+\frac{27 \Lambda_{1}^{6}}{64 u} \quad N_{f}=2: p(u)=4\left(u^{2}-\frac{\Lambda_{2}^{4}}{64}\right) \\
& N_{f}=3: p(u)=u\left(4 u-\frac{\Lambda_{3}^{2}}{64}\right) .
\end{aligned}
$$

Using the leading behavior of $F^{(0)}$ and $F_{D}^{(0)}$ from the 1-loop $\beta$-function and analytic continuation we find $\Pi=\left(\begin{array}{c}a \\ a_{D}\end{array}\right)$ as linear combination of the solutions to (2.9). We will set the dynamical scales $\Lambda_{1}=2^{\frac{2}{3}}, \Lambda_{2}=2, \Lambda_{3}=4$ in order to match the convention in the instanton counting calculations in $[4,5]$.

While in the pure $\mathrm{SU}(2)$ gauge there is a $Z_{2}$ symmetry acting on the $u$ plane, the discrete symmetries of the $u$ plane in $N_{f}=1,2,3$ cases are $Z_{3}$ symmetry, $Z_{2}$ symmetry and no symmetry respectively $[7,8]$. As we will see, these different discrete symmetries acting on the $u$ plane in the three cases $N_{f}=1,2,3$ play significant role in determining the qualitative features of the solutions. We will find the structure of $N_{f}=2$ solution closely resembles that of the case of pure gauge theory in [1], while the cases $N_{f}=1,3$ have some different qualitative features respectively.

In the next section we will review the direct integration approach for solving $F^{(g)}$. Thereafter we discuss the $N_{f}=1,2,3$ cases one by one.

\subsection{Topological string amplitude $F^{(g)}$ as polynomials of $\hat{E}_{2}$}

The main goal is to solve the topological sector of the theory and give in particular the $F^{g}(u)$ everywhere in the Coulomb moduli space. To this end we first extend the direct integration method of the holomorphic anomaly equations to the $\mathrm{SU}(2)$ gauge theory in this section to the case with massless flavors and in section 3 to the case with massive flavors. This approach was applied to $N_{f}=0$ in [1] and solved the theory completely using the gap condition. The point is to show this for theories with flavors as well.

The holomorphic anomaly equations of [21] read

$$
\begin{aligned}
\partial_{a} \partial_{\bar{a}} F^{(1)} & =\frac{1}{2} C_{a a a} C_{\bar{a}}^{a a}, \\
\bar{\partial}_{\bar{a}} F^{(g)} & =\frac{1}{2} C_{\bar{a}}^{a a}\left(D_{a} D_{a} F^{g-1}+\sum_{g=1}^{g-1} D_{a} F^{(g-h)} F^{(h)}\right), \quad \text { for } g>1 .
\end{aligned}
$$

Here we used the coordinate $a$ introduced in the last section, but the equations are of course covariant. We further introduced the Yukawa triple coupling and the connection $D_{a}$, whose calculation from the solutions of the Picard Fuchs equation are discussed below and more generally in section (5.1). First $F^{(0)}(a)$ follows from the solution $\Pi$ to (2.9) via (2.4) up to an irrelevant constant. We define then the three point Yukawa coupling as

$$
C_{a a a}=\frac{\partial^{3} F^{(0)}}{\partial a^{3}}=-2 \pi i m_{0} \frac{d \tau}{d a}=: \xi
$$


Our normalization convention is $m_{0}=2,1,1, \frac{1}{2}$ for the cases of $N_{f}=0,1,2,3$. Mathematically $\tau$ is the modular parameter and physically

$$
\tau=-\frac{1}{2 \pi i m_{0}} \frac{\partial^{2} F^{(0)}}{\partial a^{2}}
$$

a combination ${ }^{6}$ of the gauge coupling and the theta angle $\tau=\frac{\theta}{\pi}+i \frac{8 \pi}{g^{2}}$. Note that $\tau_{2}=$ $\operatorname{Im}(\tau)=\frac{\tau-\bar{\tau}}{2 i}$ multiplies the kinetic term of the vector multiplet. ${ }^{7}$ With the methods described in section 5 of [34] one can prove the following modular expression for $\xi$

$$
\begin{aligned}
& N_{f}=0: \quad \xi=\frac{8 \theta_{2}^{2}}{\theta_{3}^{4} \theta_{4}^{4}}, \quad N_{f}=1: \quad \xi=\frac{4 \sqrt{6} E_{4}^{\frac{1}{2}}\left(E_{4}^{\frac{3}{2}}-E_{6}\right)^{\frac{1}{6}}}{(-1)^{\frac{1}{6}}\left(E_{4}^{\frac{3}{2}}+E_{6}\right)}, \\
& N_{f}=2: \quad \xi=\frac{4 \theta_{2}^{2}}{\theta_{3}^{4} \theta_{4}^{4}}, \quad N_{f}=3: \quad \xi=\frac{8 \theta_{2}^{2}}{\theta_{3}^{4} \theta_{4}^{4}},
\end{aligned}
$$

where $E_{k}$ and $\theta_{k}$ are the standard Eisenstein series and Jacobi $\theta$ functions in the conventions [34]. Further the Weil-Petersson metric in the coordinate $a$ is given by

$$
G_{a \bar{a}}=2 \partial_{a} \partial_{\bar{a}} \operatorname{Re}\left(\bar{a} \partial_{a} F^{(0)}\right)=4 m_{0} \pi \tau_{2} .
$$

The connection $D_{a}$ comes entirely ${ }^{8}$ from the metric $G_{a \bar{a}}$, and is

$$
\Gamma_{a a}^{a}=\left(G_{a \bar{a}}\right)^{-1} \partial_{a}\left(G_{a \bar{a}}\right)=-\frac{i}{2 \tau_{2}} \frac{\partial \tau}{\partial a} .
$$

Note that $\Gamma_{a a}^{a}$ vanishes in the holomorphic limit $\bar{\tau} \rightarrow \infty$, confirming that the period $a$ is a flat coordinate in this limit. Finally we have

$$
C_{\bar{a}}^{a a}=G^{a \bar{a}} G^{a \bar{a}} \bar{C}_{\bar{a} \bar{a} \bar{a}} .
$$

In the following it will be important to keep track of the anti-holomorphic dependence $\Gamma_{a a}^{a}$ in $(2.11)$ in order to recover the full $F^{(g)}(\tau, \bar{\tau})$ including its anti-holomorphic dependence.

The holomorphic anomaly equation determines $F^{(g)}$ from lower genus data up to a holomorphic anomaly, which can be fixed by modularity and the gap condition. Let us start with genus one, which is somewhat special. It follows from (2.12), (2.15), (2.17) that the right hand side of the $g=1$ equation in $(2.11)$ is $\frac{1}{8 \tau_{2}^{2}}\left|\frac{\partial \tau}{\partial a}\right|^{2}$. This can be integrated to

$$
F^{(1)}=-\frac{1}{2} \log \left(\tau_{2}\right)-\log |\Phi(\tau)|^{2},
$$

where $\Phi(\tau)$ is modular form of weight $\frac{1}{2}$, which vanishes at the discriminant of the elliptic curve. In the simplest cases, e.g. $N_{f}=0$ one $\Phi(\tau)$ can be identified with the Dedekind

\footnotetext{
${ }^{6}$ Here we used the normalization of [8].

${ }^{7}$ The key requirement that the latter has to be positive suggested the occurrence of Riemann surfaces in this context, where $\tau_{2}$ is manifestly positive.

${ }^{8}$ In the global Calabi-Yau case there is an additional Kähler connection, as explained in section 5.1.
} 
$\eta$-function. Note that the transformation of $\Phi(\tau)$ as weight $\frac{1}{2}$ modular form cancels the transformation of the $-\frac{1}{2} \log \left(\tau_{2}\right)$ term. In general the form $\Phi(\tau)$ is determined by its modularity and its leading logarithmic behavior near $a_{D}^{(k)}=0$. It has been pointed out in [35] that $\log \left(a_{D}^{(k)}\right)$ comes from the gravitational one-loop $\beta$ function and its prefactor is entirely determined by the massless spectrum at the critical point.

$F^{(1)}(\tau, \bar{\tau})$ is an almost holomorphic modular function or form of weight $0^{9}$ and its $\tau$ derivative, which appears in (2.11), is an almost holomorphic form of weight 2. Modularity implies that this derivative contains the unique almost holomorphic modular weight two form

$$
\hat{E}_{2}(\tau, \bar{\tau})=E_{2}(\tau)-\frac{3}{\pi \tau_{2}},
$$

where $E_{2}(\tau)$ is the holomorphic quasimodular second Eisenstein form [34]. Under modular transformations $\tau \mapsto \tau_{\gamma}=\frac{a \tau+b}{c \tau+d}$ with $\gamma \in \Gamma_{1}=\operatorname{PSL}(2, \mathbb{Z}) E_{2}$ transforms with an inhomogeneous shift

$$
E_{2}\left(\tau_{\gamma}\right)=(c \tau+d)^{2} E_{2}(\tau)-\frac{6 i c}{\pi}(c \tau+d) .
$$

This shift cancels the shift transformation of $-\frac{3}{\pi \tau_{2}}$, so that $\hat{E}_{2}$ transforms indeed as a honest weight two form.

In calculating the right hand side of (2.11) one needs derivatives of modular forms of even positive weight. The covariant derivative $D$ in (2.11) written in terms of the $\tau$ coordinate is the so called Mass derivative

$$
\hat{D}_{\tau}=\hat{\partial}_{\tau}-\frac{k}{4 \pi \tau_{2}}
$$

Here $\hat{\partial}_{\tau}=\frac{1}{2 \pi i} \frac{d}{d \tau}$ and $k$ is the modular weight of the object acted on. The Mass derivative $\hat{D}_{\tau}$ has the important property that it maps almost holomorphic modular forms of weight $k$ into almost holomorphic modular forms of weight $k+2$. Modular invariance implies that each covariant derivative increases the leading power of $\hat{E}_{2}$ by one and all powers of $\frac{1}{\tau_{2}}$ must combine with $E_{2}(\tau)$ to form the shift invariant combination $\hat{E}_{2}(\tau, \bar{\tau})$. From this follows the important fact that for $g \geq 2$ all anholomorphic dependence of $F^{(g)}$ is in $\hat{E}_{2}$ and we can replace

$$
\frac{d}{d \bar{\tau}}=\frac{d \hat{E}_{2}}{d \bar{\tau}} \frac{d}{d \hat{E}_{2}}=\frac{3 i}{2 \pi \tau_{2}^{2}} \frac{d}{d \hat{E}_{2}}
$$

Furthermore we find that the anti-holomorphic derivative in (2.11) combines with the three point function as

$$
\frac{2 \partial_{\bar{a}} F^{(g)}}{C_{\bar{a}}^{a a}}=24 m_{0} \frac{d F^{(g)}}{d \hat{E}_{2}}, \quad \text { for } g \geq 2,
$$

so that $(2.11)$ can be written as

$$
24 m_{0} \frac{d F^{(g)}}{d \hat{E}_{2}}=D_{a}^{2} F^{(g-1)}+\sum_{r=1}^{g-1} \partial_{a} F^{(r)} \partial_{a} F^{(g-r)} .
$$

\footnotetext{
${ }^{9}$ In slight abuse of notation we indicate almost holomorphic objects by writing a $\bar{\tau}$ dependence.
} 
Since the period $a$ is a quasimodular object of weight 1 and $\hat{E}_{2}$ is of weight 2 one concludes that all $F^{(g)}$ have modular weight zero. Combining the above facts it follows that the $F^{(g)}$ are inhomogeneous polynomials of degree $3(g-1)$ in $\hat{E}_{2}$ whose coefficients are holomorphic forms of negative weight so that $F^{(g)}$ have weight zero. Defining the following derivatives

$$
\begin{aligned}
\partial_{a} F^{(r)} & =-\frac{\xi}{m_{0}} \hat{\partial}_{\tau} F^{(r)}=-\frac{\xi}{m_{0}} \hat{D}_{\tau} F^{(r)} \\
D_{a}^{2} F^{(r)} & =\left(\partial_{a}-\Gamma_{a a}^{a}\right) \partial_{a} F^{(r)}=\frac{1}{m_{0}^{2}} \xi \hat{D}_{\tau}\left(\xi \hat{D}_{\tau} F^{(r)}\right),
\end{aligned}
$$

where we used the connection (2.16) and the fact that $F^{(r)}$ and $\partial_{a} F^{(r)}$ have modular weight zero and -1 respectively, we write the holomorphic anomaly equation as

$$
24 m_{0}^{3} \frac{d F^{(g)}}{d \hat{E}_{2}}=\xi^{2}\left(\hat{D}_{\tau}^{2} F^{(g-1)}+\frac{\hat{D}_{\tau} \xi}{\xi} \hat{D}_{\tau} F^{(g-1)}+\sum_{r=1}^{g-1} \hat{D}_{\tau} F^{(r)} \hat{D}_{\tau} F^{(g-r)}\right) .
$$

This provides an unifying description of the Seiberg-Witten theory with various number of massless flavors, depending only on $m_{0}$ and the holomorphic modular form of weight -3 given in (2.14).

In each integration step the coefficients of all nonzero powers of $\hat{E}_{2}$ are determined by (2.11), while an holomorphic ambiguity of modular weight zero can be added. Boundary conditions and modularity imply that this can be always written as $\xi^{2 g-2}$ times a modular form of weight $6(g-1)$. This reduces problem of fixing the ambiguity to the determination of a finite number of terms. The remaining task is solved by an analysis of the local form of the effective action, which we discuss next.

A key concept in the analysis of effective action [8] is its transformation property under the modular group $\Gamma_{N_{f}}$ and the concept of local holomorphic coordinates in which the effective action is expanded near the critical points of the theory, where particles become massless [8]. In particular in the asymptotically free region of the gauge theory $a$ or more precisely $\frac{1}{a}$ is the correct small expansion parameter, while near points where a dyon of magnetic charge and electric charge $\left(q_{m}, q_{e}\right)$ in an $N=2$ hypermultiplet becomes massless, i.e. close to the components of the discriminant locus of $(2.2), a_{D}^{(k)}=q_{m}^{(k)} a_{D}+q_{e}^{(k)} a$ is the small expansion parameter. In most cases, i.e. for $N_{f}=0,1,2$ the $Z_{2}, Z_{3}$ and $Z_{2}$ symmetry of the theories respectively relates the dyon points and the local expansions are the same, but for $N_{f}=3$ we find truly inequivalent dyon points.

At the magnetic monopole point, also called conifold point, the leading term of the topological string amplitudes in the variable $a_{D}$, is determined by the $c=1$ string at the selfdual radius [36]. The $2 g-1$ sub-leading terms are absent. This gap structure ${ }^{10}$

$$
F^{(g) D}=\frac{c^{g-1} B_{2 g}}{2 g(2 g-2) a_{D}^{2 g-2}}+\mathcal{O}\left(a_{D}^{0}\right) .
$$

has been observed in [1] and as explained in [37] it originates indeed from integrating out a single massless hypermultiplet in the Schwinger loop contribution to the higher derivative

\footnotetext{
${ }^{10}$ Below we can always rescale $a_{D}$ so that $c=1$. The Bernoulli numbers $B_{k}$ are defined by $\sum_{k=0}^{\infty} \frac{B_{k} x^{k}}{k !}=$ $\frac{x}{e^{x}-1}$, i.e. $B_{2}=\frac{1}{6}, B_{4}=-\frac{1}{30}, B_{6}=\frac{1}{42}$ etc.
} 
effective action, which arises from a singular Calabi-Yau 3 fold with a shrinking $S^{3}$. The shrinking occurs at the conifold in the complex moduli space. The hypermultiplet in question is identified with a single dyon state in the gauge theory limit. In the gauge theory limit the compact part of the singular 3-fold becomes a nodal Riemann surface and it can be explicitly seen using the local limit of the periods around the $S^{3}$ and its dual cycle that the local limit preserves the gap condition. Indeed the gap was used already in [1] to fix the holomorphic ambiguity in the calculation of the gravitational corrections in pure SU(2) Seiberg-Witten theory. Here we find as expected that the gap occurs at all points in the moduli space of the Riemann surface where a single dyon becomes massless and a nodal singularity develops. Using the gaps and the leading coefficients we are able to fix in the $N_{f}=0,1,2,3$ cases the holomorphic ambiguity genus for all genus, and obtain exact formulae for the gravitational corrections $F^{(g)}$ that sum up all instanton contributions at each genus $g$. Mathematically one could prove the gap condition eventually in general from properties of the Nekrasov expansion and the modular transformation of the $\mathcal{F}_{g}$. We have not done this here but, merely checked the compatibility of this expansion with the gap condition in our examples.

Let us finish this section with some comments on (2.26) and a calculation of the leading terms in $\hat{E}_{2}$. First we note that the equations leading to (2.26) are invariant under the change $\hat{\partial}_{\tau}, E_{2} \rightarrow \hat{D}_{\tau}, \hat{E}_{2}$, one may therefore as well take a "holomorphic limit" and replace $\hat{D}_{\tau}, \hat{E}_{2}$ with $\hat{\partial}_{\tau}, E_{2}$ in equation (2.26), without losing any information. Furthermore the holomorphic anomaly equation (2.26) provides a very efficient way to compute topological string amplitudes. While in the Feynman rule approach of BCOV the number of diagrams grows exponentially with $g$, in the direct integration approach the number of terms in $F^{(g)}$ grows only with a power law with the genus $g$. This is similar as in the case of quintic Calabi-Yau three-fold studied in [25, 37].

The leading coefficients of $F^{(g)}$ as polynomials of $\hat{E}_{2}$ do not depend on the holomorphic ambiguity and can be computed to very high orders. Suppose we denote the leading terms by ${ }^{11}$

$$
F^{(g)}=\frac{A^{(g)}}{(g-1)\left(1152 m_{0}^{3}\right)^{g-1}} \xi^{2(g-1)} \hat{E}_{2}^{3(g-1)}+\cdots
$$

where $\cdots$ denotes terms with lower powers of $\hat{E}_{2}$. One can see that the coefficients $A^{(g-1)}$ do not depend on the holomorphic ambiguities, since there is no $E_{2}$ in the holomorphic ambiguities in all the models we study. Using the holomorphic anomaly equation (2.26), we find a simple recursion relation for all the cases $N_{f}=0,1,2,3$ of Seiberg-Witten theory

$$
\begin{aligned}
& A^{(2)}=\frac{5}{36}, \\
& A^{(g)}=(g-1) A^{(g-1)}+\sum_{r=2}^{g-2} A^{(r)} A^{(g-r)}, g>2
\end{aligned}
$$

The first few coefficients $A^{(g)}$ are $\frac{5}{36}, \frac{5}{18}, \frac{1105}{1296}, \frac{565}{162}, \cdots$.

In the next three subsections we discuss the massless $N_{f}=1,2,3$ cases one by one.

\footnotetext{
${ }^{11}$ The numerical factor 1152 is included to keep the recursion for the $A^{(g)}$ below simpler.
} 
$2.2 \quad N_{f}=1$

For the $N_{f}=1$ theory the discriminant is according to $(2.9)$

$$
\Delta_{1}=16\left(16 u^{3}+27\right) .
$$

The solution of the Picard-Fuchs equation in the weak coupling limit $u \rightarrow \infty$ is

$$
\begin{aligned}
a & =\sqrt{u}\left(1+\frac{3}{64 u^{3}}-\frac{315}{16384 u^{6}}+\frac{15015}{1048576 u^{9}}+\cdots\right) \\
a_{D} & =3 a \log (u)+\sqrt{u}\left(\frac{3}{32 u^{3}}-\frac{297}{16384 u^{6}}+\frac{9047}{1048576 u^{9}}+\cdots\right)
\end{aligned}
$$

The prepotential and gauge coupling $\tau$ are then determined by $\frac{\partial F^{(0)}}{\partial a}=a_{D}$ and $\tau=$ $-\frac{1}{2 \pi i} \frac{\partial^{2} F^{(0)}}{\partial a^{2}}$. The modulus $u$ can be expressed in terms of $\tau$ as [32]

$$
\begin{aligned}
u & =\frac{1}{8}\left(-E_{4}(\tau)^{3}-E_{6}(\tau) E_{4}(\tau)^{\frac{3}{2}}\right)^{\frac{1}{3}} \eta(\tau)^{-8} \\
& =\frac{3}{2} m^{2} \frac{E_{4}^{\frac{1}{2}}}{\left(E_{4}^{\frac{3}{2}}-E_{6}\right)^{\frac{1}{3}}}
\end{aligned}
$$

where $m$ is a one-sixth root of -1 , i.e. $m^{6}=-1$.

The holomorphic limit of the genus one amplitude is $[1,11]$

$$
F^{(1)}=-\frac{1}{2} \log \left(\frac{d a}{d u}\right)-\frac{1}{12} \log \left(\Delta_{1}\right) .
$$

This follows from $F^{(1)}=-\frac{1}{2} \log \left(G_{u \bar{u}}|\Delta|^{\frac{1}{6}}\right)$, which satisfies (2.11), in the holomorphic limit.

To provide modular formulas for all expressions we rewrite the Picard-Fuchs equation for $N_{f}=1$ in $(2.9)$ as

$$
\left(4 u^{2}+\frac{27}{4 u}\right)\left[\left(\frac{d^{2} a}{d \tau^{2}}\right) /\left(\frac{d u}{d \tau}\right)^{2}-\left(\frac{d a}{d \tau}\right)\left(\frac{d^{2} u}{d \tau^{2}}\right) /\left(\frac{d u}{d \tau}\right)^{3}\right]+a=0
$$

Using (2.32) we can obtain a differential equation for period $a$ in terms of $\tau$. In the weak coupling limit $\tau \rightarrow i \infty$, the modulus $u$ goes like $u \sim\left(E_{4}^{\frac{3}{2}}-E_{6}\right)^{-\frac{1}{3}}$, and the period $a$ goes like $a \sim \sqrt{u} \sim\left(E_{4}^{\frac{3}{2}}-E_{6}\right)^{-\frac{1}{6}}$. After fixing the normalization, it follows that the solution of (2.34) that corresponds to the period $a$ is

$$
a=\sqrt{\frac{3}{2}} \frac{m}{2} \frac{E_{4}^{\frac{1}{2}}+E_{2}}{\left(E_{4}^{\frac{3}{2}}-E_{6}\right)^{\frac{1}{6}}} .
$$

As expected the period $a$ has formally modular weight one, since $\tau=-\frac{1}{2 \pi i} \frac{\partial^{2} F^{(0)}}{\partial a^{2}}$ and $F^{(g)}$, $\frac{d}{d \tau}$ have modular weight zero, two respectively.

The holomorphic genus one amplitude $F^{(1)}$, i.e. $-\log (\Phi(\tau))$ is then

$$
F^{(1)}=-\frac{1}{12} \log \left(E_{4}^{\frac{3}{2}}+E_{6}\right) .
$$


We integrate the holomorphic anomaly equation (2.26) and expand $F^{(g)}$ around the discriminant points $\Delta_{1}(u)=0$, in order to use the gap structure. The 3 discriminant points $\Delta_{1}(u)=0$ are related by a $Z_{3}$ symmetry so we only need to consider the dual expansion around one of the 3 points. According to [8] theses points should be related to the weak coupling limit $\tau \rightarrow+i \infty$ by an S-duality transformation $\tau \rightarrow-\frac{1}{\tau}$. The Eisenstein series $E_{n}$ transform with modular weight $n$, and a shift for $E_{2}$, i.e. $E_{2} \rightarrow \tau^{2}\left(E_{2}+\frac{12}{2 \pi i \tau}\right), \quad E_{4} \rightarrow$ $\tau^{4} E_{4}, \quad E_{6} \rightarrow \tau^{6} E_{6}$. In the weak coupling limit $\tau^{2}$ is negative, so we find that under a Sduality transformation, $E_{2}, E_{6}$ change sign and $E_{4}$ doesn't. Following the approach in [1], we can find the dual period $a_{D}$ and $F_{D}^{(g)}$ by replacing $E_{2}, E_{6}$ with $-E_{2},-E_{6}$ in (2.35)

$$
a_{D}=\sqrt{\frac{3}{2}} \frac{m}{2} \frac{E_{4}^{\frac{1}{2}}-E_{2}}{\left(E_{4}^{\frac{3}{2}}+E_{6}\right)^{\frac{1}{6}}} .
$$

Note that the modulus $u$ transforms as

$$
u=\frac{3}{2} m^{2} \frac{E_{4}^{\frac{1}{2}}}{\left(E_{4}^{\frac{3}{2}}-E_{6}\right)^{\frac{1}{3}}} \rightarrow \frac{3}{2} m^{2} \frac{E_{4}^{\frac{1}{2}}}{\left(E_{4}^{\frac{3}{2}}+E_{6}\right)^{\frac{1}{3}}},
$$

i.e. the S-duality transforms $u$ indeed from $u=\infty$ to the $Z_{3}$ symmetric discriminant points $u=\frac{3}{2^{\frac{4}{3}}} m^{2}$.

It is now straightforward to expand the dual genus two amplitude $F_{D}^{(2)}$ in terms of the dual period $a_{D}$ in the weak coupling limit of the S-dual theory $\tau_{D}=-\frac{1}{\tau} \rightarrow+i \infty$. We use the gap condition as in the case of pure gauge theory [1] to fix the holomorphic ambiguity, and we find the genus two amplitude and its S-dual

$$
\begin{aligned}
F^{(2)}= & \frac{\left(E_{4}^{\frac{3}{2}}-E_{6}\right)^{\frac{1}{3}}}{2160 m^{2}\left(E_{4}^{\frac{3}{2}}+E_{6}\right)^{2}}\left[-25 E_{2}^{3} E_{4}+E_{2}^{2}\left(-135 E_{4}^{\frac{3}{2}}+30 E_{6}\right)\right. \\
& \left.+E_{2}\left(255 E_{4}^{2}-120 E_{4}^{\frac{1}{2}} E_{6}\right)-159 E_{4}^{\frac{5}{2}}+140 E_{4} E_{6}\right] \\
F_{D}^{(2)}= & \frac{\left(E_{4}^{\frac{3}{2}}+E_{6}\right)^{\frac{1}{3}}}{2160 m^{2}\left(E_{4}^{\frac{3}{2}}-E_{6}\right)^{2}}\left[25 E_{2}^{3} E_{4}+E_{2}^{2}\left(-135 E_{4}^{\frac{3}{2}}-30 E_{6}\right)\right. \\
& \left.-E_{2}\left(255 E_{4}^{2}+120 E_{4}^{\frac{1}{2}} E_{6}\right)-159 E_{4}^{\frac{5}{2}}-140 E_{4} E_{6}\right]
\end{aligned}
$$

The genus two space-time instanton expansion and the S-dual expansion are as follows

$$
\begin{aligned}
F^{(2)}= & \frac{1}{160 a^{2}}+\frac{9}{1024 a^{8}}-\frac{16749}{262144 a^{14}}+\frac{187215}{1048576 a^{20}} \\
& -\frac{6536606985}{17179869184 a^{26}}+\mathcal{O}\left(\frac{1}{a^{32}}\right) \\
F_{D}^{(2)}= & -\frac{1}{240 a_{D}^{2}}-\frac{221 a_{D}}{3^{\frac{1}{2}} 62208 m^{3}}+\frac{76289 a_{D}^{2}}{2^{\frac{1}{3}} 10077696 m^{4}}-\frac{1082609 a_{D}^{3}}{2^{\frac{2}{3}} 3^{\frac{1}{2}} 45349632 m^{5}}+\mathcal{O}\left(a_{D}^{4}\right) .
\end{aligned}
$$


We obtain the genus three amplitude using the gap condition at the conifold point

$$
\begin{aligned}
F^{(3)}= & \frac{\left(E_{4}^{\frac{3}{2}}-E_{6}\right)^{\frac{2}{3}}}{544320 m^{4}\left(E_{4}^{\frac{3}{2}}+E_{6}\right)^{4}}\left\{525 E_{2}^{6} E_{4}^{2}-350 E_{2}^{5}\left(19 E_{4}^{\frac{5}{2}}-5 E_{4} E_{6}\right)\right. \\
& +35 E_{2}^{4}\left(1225 E_{4}^{3}-694 E_{4}^{\frac{3}{2}} E_{6}+16 E_{6}^{2}\right)-280 E_{2}^{3}\left(637 E_{4}^{\frac{7}{2}}-546 E_{4}^{2} E_{6}+51 E_{4}^{\frac{1}{2}} E_{6}^{2}\right) \\
& +7 E_{2}^{2}\left(67221 E_{4}^{4}-75400 E_{4}^{\frac{5}{2}} E_{6}+14540 E_{4} E_{6}^{2}\right) \\
& -14 E_{2}\left(49821 E_{4}^{\frac{9}{2}}-68867 E_{4}^{3} E_{6}+20960 E_{4}^{\frac{3}{2}} E_{6}^{2}-560 E_{6}^{3}\right) \\
& \left.+\left(440325 E_{4}^{5}-720006 E_{4}^{\frac{7}{2}} E_{6}+308700 E_{4}^{2} E_{6}^{2}-22400 E_{4}^{\frac{1}{2}} E_{6}^{3}\right),\right\}
\end{aligned}
$$

which yields to lowest order in the asymptotically free region and near the conifold

$$
\begin{aligned}
F^{(3)}= & \frac{5}{2688 a^{4}}-\frac{3}{1024 a^{10}}+\frac{96453}{524288 a^{16}}-\frac{6065417}{4194304 a^{22}} \\
& +\frac{213776429067}{34359738368 a^{28}}+\mathcal{O}\left(\frac{1}{a^{34}}\right) \\
F_{D}^{(3)}= & \frac{1}{1008 a_{D}^{4}}-\frac{197 a_{D}}{2^{\frac{2}{3}} 3^{\frac{1}{2}} 165888 m^{5}}-\frac{54542723 a_{D}^{2}}{19591041024} \\
& +\frac{159862731109 a_{D}^{3}}{2^{\frac{1}{3}} 3^{\frac{1}{2}} 9873884676096 m}+\mathcal{O}\left(a_{D}^{4}\right) .
\end{aligned}
$$

The instanton expansion (2.44) agrees with Nekrasov's calculations (A.5) and makes predictions at higher instanton numbers.

\section{$2.3 \quad N_{f}=2$}

The discriminant is

$$
\Delta_{2}=\left(4 u^{2}-1\right)^{2} .
$$

That it is of fourth order in $u$ can be seen from (3.15) in a later section, where we provide the expression of the conifold divisor for generic flavor masses. The solution of the PicardFuchs equation at weak coupling limit $u \rightarrow \infty$ is

$$
\begin{aligned}
a & =\sqrt{u}\left(1-\frac{1}{64 u^{2}}-\frac{15}{16384 u^{4}}-\frac{105}{1048576 u^{6}}+\cdots\right) \\
a_{D} & =2 a \log (u)+\sqrt{u}\left(-\frac{1}{32 u^{2}}-\frac{13}{16384 u^{4}}-\frac{163}{3145728 u^{6}}+\cdots\right)
\end{aligned}
$$

The modulus $u$ can be expressed in terms of $\tau=-\frac{1}{2 \pi i} \frac{\partial^{2} F^{(0)}}{\partial a^{2}}$ as [32]

$$
u=\frac{1}{16} \frac{\eta\left(\frac{\tau}{2}\right)^{8}}{\eta(2 \tau)^{8}}+\frac{1}{2}=\frac{\theta_{4}^{4}(\tau)}{\theta_{2}^{4}(\tau)}+\frac{1}{2} .
$$

We verify the genus one amplitude satisfy the holomorphic anomaly equation

$$
F^{(1)}=-\frac{1}{2} \log \left(\frac{d a}{d u}\right)-\frac{1}{12} \log \left(\Delta_{2}\right)
$$


The period $a$ can also be written in terms of theta functions of $\tau$. We notice that the Picard-Fuchs equation for $N_{f}=2$ is very similar to the one of $N_{f}=0$ pure Seiberg-Witten theory studied in [1]. In particular, if we change the normalization $u \rightarrow \frac{u}{2}$, but leave $\tau$ and $a$ fixed, then the Picard-Fuchs equation (2.9) and the expression of $u$ (2.48) are exactly the same as that of pure SU(2) theory. So the expression of the period $a$ in terms of $\tau$ should be the same as that of [1] up to multiplicative constant. We find

$$
a=\frac{1}{3 \theta_{2}^{2}(\tau)}\left(E_{2}(\tau)+\theta_{3}^{4}(\tau)+\theta_{4}^{4}(\tau)\right) .
$$

However since $\Delta_{2}$ is the square of the $N_{f}=0$ discriminate $\Delta_{1}$. Therefore the genus one amplitude is not simply $F^{(1)}=-\log (\eta(\tau))$ as in the $N_{f}=0$ case [1] but rather

$$
F^{(1)}=-\frac{1}{3} \log \left(\frac{\theta_{3}^{2}(\tau) \theta_{4}^{2}(\tau)}{\theta_{2}(\tau)}\right)
$$

In the following we use the notation in [1] and define

$$
b:=\theta_{2}^{4}(\tau), \quad c:=\theta_{3}^{4}(\tau)=b+d, \quad d:=\theta_{4}^{4}(\tau),
$$

$h:=b+2 d$ and $X:=\frac{b}{1728 c^{2} d^{2}}$.

Under a $S$ duality transformation, the theta functions have modular weight two, and transform as $b \rightarrow-\tau^{2} d, c \rightarrow-\tau^{2} c, d \rightarrow-\tau^{2} b$. The Eisenstein $E_{2}$ is weight two and transforms with a shift (2.20). The period $a$ contains $E_{2}$ as well as $\theta_{2}^{2}$ and is therefore not modular invariant under $\Gamma(2)$. By a duality transformation followed by a holomorphic limit it is rather related to the dual period

$$
a_{D}=-\frac{i}{3 \theta_{4}^{2}}\left(E_{2}-b-c\right)
$$

Using the gap condition from dual expansion, we fix the genus two amplitude and find the space-time instanton expansion

$$
\begin{aligned}
F^{(2)}= & \frac{2}{15} X\left\{25 E_{2}^{3}-75 E_{2}^{2} h+15 E_{2}\left(13 b^{2}+22 c d\right)-h\left(137 b^{2}+8 c d\right)\right\}, \\
F^{(2)}= & \frac{7}{480 a^{2}}-\frac{7}{1024 a^{6}}-\frac{1425}{262144 a^{10}}-\frac{15717}{8388608 a^{14}} \\
& -\frac{8623029}{17179869184 a^{18}}+\mathcal{O}\left(\frac{1}{a^{22}}\right) .
\end{aligned}
$$

The S-dual of $F^{(2)}$ and its dual expansion fulfilling the gap condition are

$$
\begin{aligned}
& F_{D}^{(2)}=\frac{2}{15} X_{D}\left\{25 E_{2}^{3}-75 E_{2}^{2} h_{D}+15 E_{2}\left(13 d^{2}+22 b c\right)-h_{D}\left(137 d^{2}+8 b c\right)\right\} \\
& F_{D}^{(2)}=\frac{1}{120 a_{D}^{2}}+\frac{3 i a_{D}}{512}-\frac{33 a_{D}^{2}}{1024}-\frac{2147 i a_{D}^{3}}{20480}+\mathcal{O}\left(a_{D}^{4}\right)
\end{aligned}
$$

where $h_{D}=-d-2 b, X_{D}=-\frac{d}{1728 b^{2} c^{2}}$. 
We push the analysis to genus three using the gap condition. The genus three amplitude is

$$
\begin{aligned}
F^{(3)}=X^{2}\{ & 80 E_{2}^{6}-480 E_{2}^{5} h+48 E_{2}^{4}\left(41 b^{2}+104 c d\right)-\frac{32}{3} E_{2}^{3} h\left(646 b^{2}+685 c d\right) \\
& +\frac{16}{5} E_{2}^{2}\left(6503 b^{4}+23410 b^{2} c d+7637 c^{2} d^{2}\right) \\
& -\frac{32}{5} E_{2} h\left(5867 b^{4}+11605 b^{2} c d+671 c^{2} d^{2}\right) \\
& \left.+\frac{16}{105}\left(177293 b^{6}+787182 b^{4} c d+619233 b^{2} c^{2} d^{2}+40232 c^{3} d^{3}\right)\right\} .
\end{aligned}
$$

The space-time instanton expansion and the S-dual expansion are given below

$$
\begin{aligned}
F^{(3)}= & \frac{31}{8064 a^{4}}+\frac{5}{2048 a^{8}}+\frac{8843}{524288 a^{12}}+\frac{140721}{8388608 a^{16}} \\
& +\frac{318316439}{34359738368 a^{22}}+\mathcal{O}\left(\frac{1}{a^{26}}\right) \\
F_{D}^{(3)}= & \frac{1}{504 a_{D}^{4}}+\frac{45 i a_{D}}{16384}-\frac{279 a_{D}^{2}}{8192}-\frac{745933 i a_{D}^{3}}{3670016}+\mathcal{O}\left(a_{D}^{4}\right) .
\end{aligned}
$$

The instanton expansion (2.59) agree with (A.6) and make predictions at higher instanton numbers.

\section{$2.4 \quad N_{f}=3$}

The discriminant is

$$
\Delta_{3}=\frac{u}{4}(16 u-1)
$$

The solution of the Picard-Fuchs equation at weak coupling limit $u \rightarrow \infty$ is

$$
\begin{aligned}
a & =\sqrt{u}\left(1-\frac{1}{64 u}-\frac{3}{16384 u^{2}}-\frac{5}{1048576 u^{3}}+\cdots\right) \\
a_{D} & =a \log (u)+\sqrt{u}\left(-\frac{1}{32 u}-\frac{1}{16384 u^{2}}+\frac{1}{3145728 u^{3}}+\cdots\right) .
\end{aligned}
$$

In the case $N_{f}=3$, it turns out to be convenient to define the gauge coupling as $\tau=$ $-\frac{1}{\pi i} \frac{\partial^{2} F^{(0)}}{\partial a^{2}}+1$. The modulus $u$ can be expressed in terms of $\tau$ as [32]

$$
u=-\frac{1}{256} \frac{\eta\left(\frac{(\tau-1)}{2}\right)^{8}}{\eta(2(\tau-1))^{8}}=\frac{1}{256} \frac{\eta\left(\frac{\tau}{2}\right)^{8}}{\eta(2 \tau)^{8}}+\frac{1}{16}
$$

We verify that the genus one amplitude satisfy the holomorphic anomaly equation

$$
F^{(1)}=-\frac{1}{2} \log \left(\frac{d a}{d u}\right)-\frac{1}{12} \log \left(\Delta_{3}\right)-\frac{1}{4} \log (u) .
$$

We note an additional singularity at $u=0$ besides the $\Delta_{3}=0$. 
We can also use the results from pure $\mathrm{SU}(2)$ case [1] to write period $a$ and $F^{(1)}$ as theta functions of $\tau$. We notice that under a change of variable $u=\frac{\tilde{u}+1}{32}$, the Picard-Fuchs equation (2.9) and the formula (2.63) become the same as the pure gauge theory case, namely we have

$$
\begin{aligned}
& 4\left(\tilde{u}^{2}-1\right) \frac{d^{2} a}{d \tilde{u}^{2}}+a=0 \\
& \tilde{u}=\frac{1}{8} \frac{\eta\left(\frac{\tau}{2}\right)^{8}}{\eta(2 \tau)^{8}}+1=\frac{\theta_{3}^{4}(\tau)+\theta_{4}^{4}(\tau)}{\theta_{2}^{4}(\tau)} .
\end{aligned}
$$

So we can use the result of pure $\mathrm{SU}(2)$ gauge theory and we find

$$
a=\frac{1}{12 \theta_{2}^{2}(\tau)}\left(E_{2}(\tau)+\theta_{3}^{4}(\tau)+\theta_{4}^{4}(\tau)\right)
$$

and the genus one amplitude

$$
F^{(1)}=-\frac{1}{3} \log \left(\frac{\theta_{3}^{4}(\tau) \theta_{4}(\tau)}{\theta_{2}^{2}(\tau)}\right) .
$$

In the case of $N_{f}=3$, there are two different dual expansions: one at $u=0$ and one at $u=\frac{1}{16}$. Unlike the case in $N_{f}=0$ and $N_{f}=2$, the two expansions are not related by a $Z_{2}$ symmetry. The S-duality transformation transforms $u=\infty$ to $u=\frac{1}{16}$ or $\tilde{u}=1$, while a $\mathrm{T}$ and $\mathrm{S}$ duality transforms $u=\infty$ to $u=0$ or $\tilde{u}=-1$. The corresponding actions on $a, b$ and $c$ are

$$
\begin{aligned}
\text { S-duality : } & b \rightarrow-\tau^{2} d, c \rightarrow-\tau^{2} c, d \rightarrow-\tau^{2} b \\
\text { TS-duality }: & b \rightarrow \tau^{2} d, c \rightarrow-\tau^{2} b, d \rightarrow-\tau^{2} c .
\end{aligned}
$$

and yield the dual periods as

$$
\begin{aligned}
\text { S-duality : } & a_{D 1}=-\frac{i}{12 \theta_{4}^{2}}\left(E_{2}-b-c\right) \\
\text { TS-duality : } & a_{D 2}=-\frac{1}{12 \theta_{4}^{2}}\left(E_{2}-b-c\right) .
\end{aligned}
$$

It turns out there are gap structures in the dual series expansions at both $u=0$ and $u=\frac{1}{16}$, where the first sub-leading terms of the dual series at $u=0$ and $u=\frac{1}{16}$ go like constant and $a_{D}^{3}$ respectively. We are able to use this structure to fix the genus two amplitude

$$
\begin{aligned}
F^{(2)}= & \frac{b}{810 c^{2} d^{2}}\left[50 E_{2}^{3}-90 E_{2}^{2}(b+4 d)+30 E_{2}\left(2 b^{2}-4 b d+35 d^{2}\right)\right. \\
& \left.-\left(16 b^{3}+51 b^{2} d-1428 b d^{2}+443 d^{3}\right)\right] .
\end{aligned}
$$

The space-time instanton expansion and the dual expansions are

$$
\begin{aligned}
F^{(2)}= & \frac{11}{480 a^{2}}+\frac{5}{1024 a^{4}}-\frac{109}{262144 a^{6}}+\frac{83}{4194304 a^{8}} \\
& -\frac{13361}{1717869184 a^{10}}+\mathcal{O}\left(\frac{1}{a^{12}}\right)
\end{aligned}
$$




$$
\begin{aligned}
& F_{D 1}^{(2)}=\frac{1}{240 a_{D 1}^{2}}+\frac{1}{2}+\frac{271 i a_{D 1}}{16}-\frac{3811 a_{D 1}^{2}}{16}-\frac{50781 i a_{D 1}^{3}}{20}+\mathcal{O}\left(a_{D 1}^{4}\right) \\
& F_{D 2}^{(2)}=\frac{1}{15 a_{D 2}^{2}}+\frac{4 a_{D 2}^{3}}{5}-\frac{75 a_{D 2}^{4}}{4}+\frac{2155 a_{D 2}^{5}}{8}+\mathcal{O}\left(a_{D 2}^{6}\right)
\end{aligned}
$$

The vanishing of the subleading coefficients in both of the dual series expansions up order $a_{D 1}^{0}$ and $a_{D 2}^{3}$ respectively yield independent conditions. These enable us to precisely to fix the unknowns in the ansatz for the holomorphic ambiguity, which is increased w.r.t. to the $N_{f}=0$ and $N_{f}=2$ case, because there is no $Z_{2}$ symmetry $u \rightarrow-u$ in the $N_{f}=3$ theory.

We push the analysis to genus three

$$
\begin{aligned}
F^{(3)}= & \frac{2 b^{2}}{76545 c^{4} d^{4}}\left\{1050 E_{2}^{6}-1050 E_{2}^{5}(5 b+13 d)+210 E_{2}^{4}\left(80 b^{2}+233 b d+374 d^{2}\right)-\right. \\
& 70 E_{2}^{3}\left(529 b^{3}+1752 b^{2} d+1731 b d^{2}+3764 d^{3}\right)+42 E_{2}^{2}\left(1172 b^{4}+3985 b^{3} d+\right. \\
& \left.4563 b^{2} d^{2}-9299 b d^{3}+12818 d^{4}\right)-42 E_{2}\left(844 b^{5}+3109 b^{4} d+4765 b^{3} d^{2}+\right. \\
& \left.12404 b^{2} d^{3}-63022 b d^{4}+9554 d^{5}\right)+\left(10718 b^{6}+44304 b^{5} d+81507 b^{4} d^{2}+\right. \\
& \left.\left.19406 b^{3} d^{3}+2674506 b^{2} d^{4}-2382348 b d^{5}+117557 d^{6}\right)\right\}
\end{aligned}
$$

The space-time instanton expansion and the two dual expansions are

$$
\begin{aligned}
F^{(3)}= & \frac{47}{8064 a^{4}}-\frac{1}{512 a^{6}}+\frac{769}{534288 a^{8}}-\frac{1595}{8388608 a^{10}} \\
& +\frac{506627}{34359738368 a^{12}}+\mathcal{O}\left(\frac{1}{a^{14}}\right), \\
F_{D 1}^{(3)}= & \frac{1}{1008 a_{D 1}^{4}}+10+\frac{29327 i a_{D 1}}{32}-\frac{413345 a_{D 1}^{2}}{16}+\mathcal{O}\left(a_{D 1}^{3}\right), \\
F_{D 2}^{(3)}= & \frac{4}{63 a_{D 2}^{4}}-\frac{90 a_{D 2}^{3}}{7}+\frac{4183 a_{D 2}^{4}}{8}-\frac{35483 a_{D 2}^{5}}{3}+\mathcal{O}\left(a_{D 2}^{6}\right),
\end{aligned}
$$

where the space-time instanton expansion (2.72) again agrees with Nekrasov's calculations (A.7) and makes predictions at higher instanton numbers. Note that the non-zero sub-leading term of the two dual series appear at order $a_{D 1}^{0}$ and $a_{D 2}^{3}$ respectively, as the genus two case.

\section{$3 \quad \mathrm{SU}(2)$ Seiberg-Witten theory with massive hypermultiplets}

In this section, we will show that the gravitational couplings $F^{(g)}$ for the massive SeibergWitten theory can be solved as polynomials of generators of the relevant modular functions, whose coefficients are rational functions of the Coulomb modulus $u$ as well as the mass parameters $m_{i}$. The equation $J(\tau)=R_{N_{F}}(u, \underline{m})$ still governs the occurrence of the modular functions and relevant group is again the quotient of $\operatorname{PSL}(2, \mathbb{Z})$ by the permutation group acting on the roots $u(J, \underline{m})$. Mathematically the mass parameters $m_{i}$ are known as isomonodromic deformation parameters.

\subsection{The prepotential $F^{(0)}$}

For the massive case the Picard-Fuchs equation is much more complicated than the massless case. There is a standard though tedious way to derive the Picard-Fuchs equations from the 
Seiberg-Witten curve [38]. The Picard-Fuchs equations for SU(2) Seiberg-Witten theory with massive hypermultiplets were derived in $[39,40]$ and for the case of one massive hypermultiplet $\left(N_{f}=1\right)$ it is given by

$$
\begin{gathered}
\frac{d^{3} \Pi}{d u^{3}}+\frac{3 \Delta_{1}+\left(4 m_{1}^{2}-3 u\right) \frac{\partial \Delta_{1}}{\partial m_{1}}}{\Delta_{1}\left(4 m_{1}^{2}-3 u\right)} \frac{d^{2} \Pi}{d u^{2}} \\
-\frac{8\left[4\left(2 m_{1}^{2}-3 u\right)\left(4 m_{1}^{2}-3 u\right)+3\left(3 \Lambda_{1}^{3} m_{1}-4 u^{2}\right)\right]}{\Delta_{1}\left(4 m_{1}^{2}-3 u\right)} \frac{d \Pi}{d u}=0 .
\end{gathered}
$$

Here $m_{1}$ is the mass of the hypermultiplet and $\Delta_{1}$ is the discriminant of the SeibergWitten curve

$$
\Delta_{1}=256 u^{3}-256 m_{1}^{2} u^{2}-288 \Lambda_{1}^{3} m_{1} u+256 \Lambda_{1}^{3} m_{1}^{3}+27 \Lambda_{1}^{6} .
$$

The differential equation (3.1) has a second singularity at the vanishing locus of

$$
\Delta_{2}=4 m_{1}^{2}-3 u \text {. }
$$

In order to match with Nekrasov's convention [4], we again set the dynamical scale $\Lambda_{1}=2^{\frac{2}{3}}$.

In the weak coupling region near $u \rightarrow \infty$, the Picard-Fuchs equation (3.1) has a constant solutions and two other solution corresponding to the periods $a$ and $a_{D}$. It was found in [39]

$$
\begin{aligned}
a= & \sqrt{u}\left[1-\frac{m_{1}}{4 u^{2}}+\frac{3}{64 u^{3}}-\frac{15 m_{1}^{2}}{64 u^{4}}+\frac{35 m_{1}}{256 u^{5}}-\frac{105\left(3+64 m_{1}^{3}\right)}{16384 u^{6}}\right. \\
& \left.+\frac{3465 m_{1}^{2}}{8192 u^{7}}+\mathcal{O}\left(\frac{1}{u^{8}}\right)\right], \\
a_{D}= & 3 a \log (u)+\sqrt{u}\left[-\frac{m_{1}^{2}}{u}-\left(\frac{m_{1}}{2}+\frac{m_{1}^{4}}{6}\right) \frac{1}{u^{2}}+\left(\frac{3}{32}-\frac{m_{1}^{3}}{4}-\frac{m_{1}^{6}}{15}\right) \frac{1}{u^{3}}\right. \\
& \left.+\left(\frac{3 m_{1}^{2}}{64}-\frac{m_{1}^{5}}{8}-\frac{m_{1}^{8}}{28}\right) \frac{1}{u^{4}}+\mathcal{O}\left(\frac{1}{u^{5}}\right)\right] .
\end{aligned}
$$

We solved the prepotential using $\frac{\partial F^{(0)}}{\partial a}=a_{D}$ and checked it against Nekrasov's result summarized in (A.1).

There are relations between the period $a$, Coulomb modulus $u$ and the gauge coupling $\tau$, which become useful for solving the model. After a $P G L(3, \mathbb{C})$ transformation, the massive Seiberg-Witten curve can brought into Weierstrass form (2.6). For $N_{f}=1$ one has

$$
\begin{aligned}
& g_{2}\left(u, m_{1}\right)=\frac{4}{3} u^{2}-4 m_{1} \\
& g_{3}\left(u, m_{1}\right)=-\frac{8}{27} u^{3}+\frac{4}{3} m_{1} u-1 .
\end{aligned}
$$

Using the fact that the period equation is solved in terms of modular forms [41] (see [34] for an elementary review), the definition of $\tau=-\frac{1}{2 \pi i} \frac{\partial a_{D}}{\partial a}$ and the $J$ function (2.7) one can show that the period $a$ satisfies the equation [42]

$$
\frac{d u}{d a}=\sqrt{-18 \frac{g_{3}\left(u, m_{1}\right)}{g_{2}\left(u, m_{1}\right)} \frac{E_{4}(\tau)}{E_{6}(\tau)}} .
$$


Note that this equation is universal for $N_{f}=0,1,2,3$ in the sense that it depends on the specifics of the curve only via $g_{2}(u, \underline{m})$ and $g_{3}(u, \underline{m})$.

\subsection{Gravitational couplings}

We shall follow the approach in [1] and treat the period $a$ as a flat coordinate in the holomorphic limit. The singular locus of the Picard-Fuchs equation (3.1) is at $\Delta_{1}=0$ and $\Delta_{2}=0 . \Delta_{1}=0$ is the the conifold divisor, i.e. one hypermultiplet becomes massless for these values of the moduli. According to [35] each hypermultiplet contributes $-\frac{1}{12}$ to one-loop gravitational $\beta$ function, which yields a logarithmic running of the coupling of $R_{+}^{2}$. This gives rise to an $-\frac{1}{12} \log \left(\Delta_{1}\right) R_{+}^{2}$ contribution in the one loop effective action, compare (2.5). On the other hand the conformal locus lies on $\Delta_{2}=0$, as discussed further in section 4 , and here the $\beta$ function and hence the $\log \left(\Delta_{2}\right)$ contribution to $F^{(1)}$ vanishes. This fixes the ambiguity at genus zero and the holomorphic limit [11]

$$
F^{(1)}=-\frac{1}{2} \log \left(\frac{d a}{d u}\right)-\frac{1}{12} \log \left(\Delta_{1}\right)
$$

agrees with Nekrasov's calculation (A.2) up to an ambiguous constant. The form of (3.7) has been already noted in [43].

Using (3.6) the genus one gravitational correction $F^{(1)}$ can be also written as

$$
F^{(1)}=-\frac{1}{12} \log \left(\frac{g_{2}^{3}\left(g_{2}^{3}-27 g_{3}^{2}\right)}{g_{3}^{3}} \frac{E_{6}^{3}}{E_{4}^{3}}\right) .
$$

As discussed in section $(2.1)$ the $F^{(g)}(g \geq 2)$ of Seiberg-Witten theory are modular invariant with weight zero. As we have seen the covariant derivatives in the recursion lead to an anholomorphic dependence, which can be completely absorbed into powers of the nonholomorphic Eisenstein series $\hat{E}_{2}(\tau)$. Their modular transformation must be compensated by holomorphic modular forms. We claim that all anholomorphic dependence can be absorbed into the weight zero anholomorphic modular form

$$
X(\tau)=\frac{\hat{E}_{2}(\tau) E_{4}(\tau)}{E_{6}(\tau)} .
$$

This can be established by rewriting the holomorphic anomaly equation $(g \geq 2)$ as

$$
\frac{\partial F^{(g)}(X, u)}{\partial X}=\frac{E_{6}}{24 E_{4}}\left(\frac{d^{2} F^{(g-1)}}{d a^{2}}+\sum_{r=1}^{g-1} \frac{d F^{(r)}}{d a} \frac{d F^{(g-r)}}{d a}\right) .
$$

Using the relations of $a, u$ and $\tau$ in (2.7) and (3.6), and induction one can easily show the right hand side of the above equation is a polynomial of $X$ of degree $3 g-4$ whose coefficients are rational function of $u$. It follows that the higher genus $F^{(g)}(g \geq 2)$ are polynomials of $X(\tau)$ of degree ${ }^{12} 3 g-3$, whose coefficients are rational functions of the modulus $u$ and $m_{1}$.

\footnotetext{
${ }^{12}$ As we explained in the massless cases in section 2.1 , there is an isomorphism between $E_{2}$ and $\hat{E}_{2}$. So one can treat the above equations by replacing $\hat{E}_{2}$ with $E_{2}$ in the holomorphic limit.
} 
Given (3.8) it is easy to integrate (3.10) up to the holomorphic ambiguity. e.g. for genus two we find

$$
\begin{aligned}
F^{(2)}=\frac{1}{\Delta(u)^{2}}\{- & 2880\left(3 u-4 m_{1}^{2}\right)^{2}\left(\frac{g_{3} X}{g_{2}}\right)^{3}-96\left(252 u^{3}-648 m_{1}^{2} u^{2}+\right. \\
& \left.\left(352 m_{1}^{4}+54 m_{1}\right) u+27\left(8 m_{1}^{3}-9\right)\right)\left(\frac{g_{3} X}{g_{2}}\right)^{2} \\
& -\frac{64}{3}\left[324 u^{4}-528 m_{1}^{2} u^{3}+4 m_{1}\left(76 m_{1}^{3}-27\right) u^{2}\right. \\
& \left.\left.-36\left(26 m_{1}^{3}+27\right) u+3 m_{1}^{2}\left(128 m_{1}^{3}+729\right)\right]\left(\frac{g_{3} X}{g_{2}}\right)\right\}+f^{(2)},
\end{aligned}
$$

where $f^{(2)}$ is the holomorphic ambiguity, which is again a rational function of $u$ and $m_{1}$.

\subsection{Fixing the holomorphic ambiguity with dual expansions}

In order to fix the holomorphic ambiguity, we use the gap structure in the dual expansion at a discriminant point $u=u_{1}$, where $\Delta\left(u_{1}\right)=0$. The formulae are essentially the same as the semi-classical limit $u \rightarrow \infty$, and we just need to use the dual coupling and period $\tau_{D}$ and $a_{D}$ in places of $\tau, a$. The formulae (2.7), (3.6) become

$$
\begin{aligned}
J\left(\tau_{D}\right) & =\frac{g_{2}(u)^{3}}{g_{2}(u)^{3}-27 g_{3}(u)^{2}} \\
\frac{d u}{d a_{D}} & =\sqrt{-18 \frac{g_{3}(u)}{g_{2}(u)} \frac{E_{4}\left(\tau_{D}\right)}{E_{6}\left(\tau_{D}\right)}}
\end{aligned}
$$

From the above formulae we see that around the discriminant point $u \sim u_{1}$, the dual theory is indeed weakly coupled in terms of the dual coupling $\tau_{D} \rightarrow i \infty$, and the asymptotic behavior of the dual period is $a_{D} \sim\left(u-u_{1}\right)$.

We can now replace $\tau$ in the genus two formula (3.11) with dual coupling $\tau_{D}$, and expand $F^{(2) D}$ in terms of the dual period $a_{D}$.

The ambiguity $f^{(g)}$ can a priori have poles at the singular points of Picard-Fuchs equation $\Delta(u)=0$ and $\Delta_{2}=0$. However $\Delta_{2}=0$ does not appear as a pole in the holomorphic ambiguity. This is because there is a conformal massless spectrum at that locus in the moduli space, similar to the situation encountered in [37] for the Gepner point in the Calabi-Yau moduli space. The singular behavior of $f^{(g)}$ at the conifolds implies then that $f^{(g)}=\frac{p_{n}(u)}{\Delta_{1}^{2 g-2}}$, where $p_{n}(u)$ is a polynomial in $u$ of degree $n$. Since $f^{(g)}$ must be regular at $u \rightarrow \infty$ and $\Delta_{1} \sim u^{3}$ we get that $n \leq 6 g-6$. In fact it turns out that $n=6 g-7$. Using the $2 g-1$ first coefficients in (2.27) at the three inequivalent conifold points, we see that the equations following from the gap condition overdetermine the coefficients of $p_{n}(u)$. This fixes the holomorphic ambiguity for all $g$. e.g. for genus two we get

$$
\begin{aligned}
f^{(2)}= & \frac{128}{405 \Delta(u)^{2}}\left[-684 u^{5}+3192 m_{1}^{2} u^{4}-2 m_{1}\left(656 m_{1}^{3}+4293\right) u^{3}+378\left(8 m_{1}^{3}+45\right) u^{2}\right. \\
& \left.-54 m_{1}^{2}\left(80 m_{1}^{3}+183\right) u+27\left(664 m_{1}^{3}-729\right)\right] .
\end{aligned}
$$


Unlike the massless case, here the constant term in the dual expansion does not vanish. Although it is too complicated to write down the constant term, we have checked it indeed vanishes in the massless limit $m_{1}=0$.

The holomorphic ambiguity (3.14) together with (3.11) gives the exact formula for genus two $F^{(2)}$ in the massive $N_{f}=1$ Seiberg-Witten theory. We have checked the agreement with Nekrasov's instanton calculation in semi-classical limit. We can furthermore obtain predictions for higher instanton results at genus two. For example, the 6-instanton and 7-instanton results are

$$
\begin{aligned}
F_{6-\text { instanton }}^{(2)} & =\frac{1497720 a^{6}-39720651 a^{4} m_{1}^{2}+131881442 a^{2} m_{1}^{4}-96877135 m_{1}^{6}}{8388608 a^{26}} \\
F_{7-\text { instanton }}^{(2)} & =\frac{3\left(6542298 a^{6} m_{1}-73190615 a^{4} m_{1}^{3}+181612908 a^{2} m_{1}^{5}-117503791 m_{1}^{7}\right)}{8388608 a^{30}} .
\end{aligned}
$$

\subsection{Comments on the $N_{f}=2,3$ cases and integrability}

We can transform the Seiberg-Witten curve for $N_{f}=2,3$ with generic hypermultiplet masses into Weierstrass form. The conifold divisors $\Delta=g_{2}^{3}-27 g_{3}^{2}=0$ for the $N_{f}=2,3$ cases are (here we use the convention for the QCD scale $\Lambda_{N_{f}=2}=2, \Lambda_{N_{f}=3}=4$ )

$$
\begin{aligned}
\Delta_{N_{f}=2}= & 16 u^{4}-16\left(m_{1}^{2}+m_{2}^{2}\right) u^{3}+\left(16 m_{1}^{2} m_{2}^{2}-80 m_{1} m_{2}-8\right) u^{2} \\
& +\left[36\left(m_{1}^{2}+m_{2}^{2}\right)+72 m_{1} m_{2}\left(m_{1}^{2}+m_{2}^{2}\right)\right] u \\
& +1-12 m_{1} m_{2}-6 m_{1}^{2} m_{2}^{2}-64 m_{1}^{3} m_{2}^{3}-27\left(m_{1}^{4}+m_{2}^{4}\right), \\
\Delta_{N_{f}=3}= & -16 u^{5}+\left(1+16 m_{1}^{2}+16 m_{2}^{2}+16 m_{3}^{2}\right) u^{4} \\
& +\left[8\left(m_{1}^{2}+m_{2}^{2}+m_{3}^{2}\right)-16\left(m_{1}^{2} m_{2}^{2}+m_{1}^{2} m_{3}^{2}+m_{2}^{2} m_{3}^{2}\right)+88 m_{1} m_{2} m_{3}\right) u^{3} \\
& +f_{2}\left(m_{1}, m_{2}, m_{3}\right) u^{2}+f_{1}\left(m_{1}, m_{2}, m_{3}\right) u++f_{0}\left(m_{1}, m_{2}, m_{3}\right),
\end{aligned}
$$

where $f_{2}, f_{1}, f_{0}$ are some symmetric polynomial of $m_{1}, m_{2}, m_{3}$.

The number of conifold point is $n=3,4,5$ for $N_{f}=1,2,3$ and these points are distinct for generic hypermultiplet masses. The ambiguity at genus $g$ is a rational function whose denominator is $\Delta^{2 g-2}$, while regularity at $u=\infty$ constrains the number of unknown constants in the holomorphic ambiguity to be $n(2 g-2)$. The gap boundary conditions at each of the $n$ distinct conifold singularities provide $2 g-2$ conditions. This is exactly enough information to fix the holomorphic ambiguity.

We have also checked the genus one formula

$$
F^{(1)}=-\frac{1}{2} \log \left(\frac{d a}{d u}\right)-\frac{1}{12} \log (\Delta)
$$

agrees with Nekrasov's instanton counting formulae for $N_{f}=2,3$ cases for generic masses when we expand it at $u=\infty$.

\section{$4 \mathrm{SU}(2)$ Seiberg-Witten theory at superconformal points}

One of the most interesting aspect of the Seiberg-Witten solution of $N=2$ gauge theories is that it allows to study regions in the parameter spaces where previously unknown types 
of four dimensional interacting field theories have been discovered. Of particular interest are the points where several dyons become massless, which have electric as well as magnetic charges. It is well known that no effective action with only local interactions can be written down in that case and one says the massless dyons are mutually non-local. Geometrically the situation is characterized by the fact that pairs of cycles which mutually non-vanishing intersection numbers vanish. It also implies that the divisors, where mutually non-local dyons vanish, intersect in the moduli space.

In the previous section 2 we studied the case where the bare masses of hypermultiplets are zero. In this case the extra massless particles at the colliding singularities are mutually local. In [22], some special cases of hypermultiplet masses are studied where two mutually non-local singular points in the $u$ plane collide. In the case there is a non-trivial interacting superconformal field theory at the colliding singularity in the $u$ plane. Besides the known $N_{f}=4$ superconformal field theory, three new $\mathcal{N}=2$ superconformal field theories were found [22] from $\mathrm{SU}(2)$ Seiberg-Witten theory with $N_{f}=1,2,3$ flavors, and are denoted as $\left(N_{f}, 1\right)$ superconformal field theory respectively .

A technically interesting aspect is that the equation (2.7), which is for general masses, compare (3.5), not easily solvable for $u(\tau)$, becomes simple and solvable at the conformal points, which allows below to find explicit formulas for the amplitudes in terms of modular forms.

\section{1 $N_{f}=1, m_{1}=\frac{3 \Lambda_{1}}{4}$}

We follow our previous notation $\Lambda_{1}=2^{\frac{2}{3}}$. In the special case of the mass of the flavor $m_{1}=$ $\frac{3 \Lambda_{1}}{4}$, there is a non-trivial $(1,1)$ superconformal field theory at $u=\frac{3}{2^{\frac{2}{3}}}$ where two mutually non-local massless dyons collide, and there is another dyon singularity at $u=-\frac{15}{4 \cdot 2^{\frac{2}{3}}}$. The $(1,1)$ superconformal field theory at $u=\frac{3}{2^{\frac{2}{3}}}$ is equivalent to the Argyres-Douglas point originally found in pure SU(3) Seiberg-Witten theory in [44]. The discriminant is

$$
\Delta \sim\left(u-\frac{3}{2^{\frac{2}{3}}}\right)^{2}\left(u+\frac{15}{4 \cdot 2^{\frac{2}{3}}}\right) .
$$

Eq. (2.7) simplifies to

$$
\frac{4(\tilde{u}+1)^{3}(\tilde{u}-1)}{4 \tilde{u}+5}=\frac{E_{4}^{3}}{E_{6}^{2}-E_{4}^{3}}
$$

where $\tilde{u}=\frac{2^{\frac{2}{3}} u}{3}$. The equation for $u$ has 4 solutions and 3 of them have the correct asymptotic behavior $u \rightarrow \infty$ in the weak coupling limit $\tau \rightarrow i \infty$. These 3 solutions are related by a $Z_{3}$ transformation and we just need to consider one solution

$$
u=\frac{3}{2^{\frac{5}{3}}}\left(-1+\sqrt{x}+\sqrt{3-x+\frac{2 E_{6}^{2}}{\left(E_{6}^{2}-E_{4}^{3}\right) \sqrt{x}}}\right)
$$

with $x=1+\frac{E_{4}^{2}}{\left(E_{6}^{2}-E_{4}^{3}\right)^{\frac{2}{3}}}-\frac{E_{4}}{\left(E_{6}^{2}-E_{4}^{3}\right)^{\frac{1}{3}}}$. 
The formula (3.6) becomes

$$
\frac{d a}{d u}=\left(\frac{2^{\frac{2}{3}}(\tilde{u}+1)}{6\left(2 \tilde{u}^{2}+2 \tilde{u}-1\right)} \frac{E_{6}}{E_{4}}\right)^{\frac{1}{2}}
$$

and the genus one amplitude becomes

$$
\begin{aligned}
F^{(1)} & =-\frac{1}{2} \log \left(\frac{d a}{d u}\right)-\frac{1}{12} \log (\Delta) \\
& =-\frac{1}{12} \log \left(\frac{(4 \tilde{u}+5)(\tilde{u}+1)^{3}(\tilde{u}-1)^{2}}{\left(2 \tilde{u}^{2}+2 \tilde{u}-1\right)^{3}} \frac{E_{6}^{3}}{E_{4}^{3}}\right),
\end{aligned}
$$

which can be written entirely in terms of $\tau$ using (4.3).

We study the gravitational couplings of the theory at the superconformal point $u=\frac{3}{2^{\frac{2}{3}}}$ in more details. The Picard-Fuchs equation has a constant solution around this point and two power series solutions. The two power series serve as the flat coordinate $a$ and the derivative of prepotential $\frac{\partial F^{(0)}}{\partial a}$ around this point. Denoting $z=u-u_{1} \rightarrow 0$, we find the solutions are

$$
\begin{aligned}
a & =z^{\frac{5}{6}}\left(1-\frac{10}{891} 2^{\frac{2}{3}} z+\frac{128}{111537} 2^{\frac{1}{3}} z^{2}-\frac{6272}{36669429} z^{3}+\mathcal{O}\left(z^{4}\right)\right), \\
\frac{\partial F^{(0)}}{\partial a} & =z^{\frac{7}{6}}\left(1-\frac{28}{1053} 2^{\frac{2}{3}} z+\frac{400}{124659} 2^{\frac{1}{3}} z^{2}-\frac{4096}{7971615} z^{3}+\mathcal{O}\left(z^{4}\right)\right) .
\end{aligned}
$$

The scaling behavior of the period of the Picard-Fuchs equation as $a \sim z^{\frac{5}{6}}$ agrees with the analysis presented in [22]. We can invert the series and solve the prepotential in terms of the flat coordinate

$$
F^{(0)}=a^{\frac{12}{5}}-\frac{28}{3861} 2^{\frac{2}{3}} a^{\frac{18}{5}}+\frac{63872}{142457535} 2^{\frac{1}{3}} a^{\frac{18}{5}}-\frac{11006912}{256086163575} a^{6}+\mathcal{O}\left(a^{\frac{36}{5}}\right) .
$$

Here we have not fixed the normalization of prepotential and the flat coordinate $a$. As usual the prepotential is determined up to a quadratic polynomial of $a$ due to the ambiguity in choosing the basis of the solutions of the Picard-Fuchs equation.

For the genus one free energy, we find

$$
F^{(1)}=-\frac{1}{10} \log (a)-\frac{1}{891} 2^{\frac{2}{3}} a^{\frac{6}{5}}+\frac{5732}{22493295} 2^{\frac{1}{3}} a^{\frac{12}{5}}+\mathcal{O}\left(a^{\frac{18}{5}}\right) .
$$

For genus two free energy, we can use the result we derive for generic mass parameter $m_{1}$, and specialize to the superconformal point. Using formulae (3.12) we can derive the expression for the Eisenstein series

$$
\begin{aligned}
& E_{4}(\tau)=12 g_{2}\left(\frac{d a}{d u}\right)^{4}, \quad E_{6}(\tau)=-216 g_{3}\left(\frac{d a}{d u}\right)^{6}, \\
& X(\tau)=\frac{E_{2}(\tau) E_{4}(\tau)}{E_{6}(\tau)}=\frac{2 E_{4}(\tau) E_{6}(\tau)-3 E_{4}(\tau)^{3}\left(\frac{d E_{4}(\tau)}{d \tau}\right) /\left(\frac{d E_{6}(\tau)}{d \tau}\right)}{2 E_{4}(\tau) E_{6}(\tau)-3 E_{6}(\tau)^{2}\left(\frac{d E_{4}(\tau)}{d \tau}\right) /\left(\frac{d E_{6}(\tau)}{d \tau}\right)} .
\end{aligned}
$$


We find that the leading singularity at genus two is $F^{(2)}=\mathcal{O}\left(\frac{1}{z}\right)$, even though for a generic holomorphic ambiguity $f^{(2)}$ one has a leading singular behavior of $f^{(2)}=\mathcal{O}\left(\frac{1}{z^{4}}\right)$. The expansion in flat coordinate is

$$
F^{(2)}=\frac{133}{38880 a^{\frac{6}{5}}}+\frac{19}{360855 \cdot 2^{\frac{1}{3}}}-\frac{79793}{1656324450} \frac{a^{\frac{6}{5}}}{2^{\frac{2}{3}}}+\frac{4310932}{332775068175} a^{\frac{12}{5}}+\mathcal{O}\left(a^{\frac{18}{5}}\right) .
$$

This kind of singularity behavior is very similar to the situation at the orbifold singularity in compact Calabi-Yau spaces encountered in [37], where the $F^{(g)}$ turns out to be less singular than naively expected. Here the massless particles scale as the period $a \sim z^{\frac{5}{6}}$ in the limit $z \rightarrow 0$ and we know $F^{(g)}$ should be no more singular than $a^{2-2 g}$ from the usual Gopakumar-Vafa argument of integrating out charged particles in the graviphoton background. This explains the leading singularity in the expression of $F^{(2)}$ above.

\section{2 $N_{f}=2, m_{1}=m_{2}= \pm \frac{\Lambda_{2}}{2}$}

We follow our previous notation $\Lambda_{2}=2$. There is a $(2,1)$ superconformal field theory at $u=\frac{3}{2}$ where a double singularity collides with a mutually non-local dyon singularity, and there is another dyon singularity at $u=-\frac{5}{2}$. The discriminant is

$$
\Delta \sim\left(u-\frac{3}{2}\right)^{3}\left(u+\frac{5}{2}\right) \text {. }
$$

We solve a cubic equation for $u$, and there are 2 solutions with the correct asymptotic behavior in weak coupling limit. As before they are related by a $Z_{2}$ symmetry. We consider one solution

$$
\begin{aligned}
u= & -\frac{3}{2}+\frac{3 e^{-\frac{\pi}{6} i} E_{4}\left(E_{6}+i\left(E_{4}^{3}-E_{6}^{2}\right)^{\frac{1}{2}}\right)^{\frac{1}{3}}}{2\left(E_{4}^{3}-E_{6}^{2}\right)^{\frac{1}{2}}} \\
& +\frac{3 e^{\frac{\pi}{6} i} E_{4}\left(E_{6}-i\left(E_{4}^{3}-E_{6}^{2}\right)^{\frac{1}{2}}\right)^{\frac{1}{3}}}{2\left(E_{4}^{3}-E_{6}^{2}\right)^{\frac{1}{2}}} .
\end{aligned}
$$

From (3.6) one gets

$$
\frac{d a}{d u}=\frac{1}{2}\left(\frac{(2 u+3)}{(2 u-3)(u+3)} \frac{E_{6}(\tau)}{E_{4}(\tau)}\right)^{\frac{1}{2}}
$$

which allows to write the genus one amplitude as

$$
\begin{aligned}
F^{(1)} & =-\frac{1}{2} \log \left(\frac{d a}{d u}\right)-\frac{1}{12} \log (\Delta) \\
& =-\frac{1}{12} \log \left(\frac{(2 u+3)^{3}(2 u+5)}{(u+3)^{3}} \frac{E_{6}^{3}}{E_{4}^{3}}\right) .
\end{aligned}
$$

One can use the expression of $u$ in equation (4.12) to obtain a formula for genus one amplitude $F^{(1)}$ purely in terms of gauge coupling $\tau$.

There are two other identical $(2,1)$ superconformal field theories at $m_{1}=-m_{2}= \pm i \frac{\Lambda_{2}}{2}$. These are the same theories as the ones at $m_{1}=m_{2}= \pm \frac{\Lambda_{2}}{2}$, to which they are related by the transformation $u \rightarrow-u$. 
As in the $N_{f}=1$ case, we can solve the flat coordinate $a$ and express the prepotential $F^{(0)}$ and genus one free energy $F^{(1)}$ in terms of it. The best way to solve the flat coordinate is to use the Picard-Fuchs equation, since at the superconformal point it is not convenient to do perturbative expansion the Eisenstein series. The Picard-Fuchs equation for the massive $N_{f}=2,3$ Seiberg-Witten theory were found in [40]. We specialize to the case of mass $m_{1}=m_{2}= \pm \frac{\Lambda_{2}}{2}$. Denote $z=u-\frac{3}{2}$ we find the solutions of the Picard-Fuchs equation around $z=0$

$$
\begin{aligned}
a & =z^{\frac{3}{4}}\left(1-\frac{3}{224} z+\frac{25}{22528} z^{2}-\frac{9}{65536} z^{3}+\mathcal{O}\left(z^{4}\right)\right), \\
\frac{\partial F^{(0)}}{\partial a} & =z^{\frac{5}{4}}\left(1-\frac{5}{96} z+\frac{147}{26624} z^{2}-\frac{847}{1114112} z^{3}+\mathcal{O}\left(z^{4}\right)\right) .
\end{aligned}
$$

The genus zero and one free energy up to a constant are

$$
\begin{aligned}
& F^{(0)}=a^{\frac{8}{3}}-\frac{5}{252} a^{4}+\frac{3197}{2690688} a^{\frac{16}{3}}-\frac{6883}{65680384} a^{\frac{20}{3}}+\mathcal{O}\left(a^{8}\right) \\
& F^{(1)}=-\frac{1}{6} \log (a)-\frac{5}{672} a^{\frac{4}{3}}+\frac{1409}{1655808} a^{\frac{8}{3}}-\frac{11873}{92725248} a^{4}+\mathcal{O}\left(a^{\frac{16}{3}}\right) .
\end{aligned}
$$

4.3 $N_{f}=3, m_{1}=m_{2}=m_{3}=\frac{\Lambda_{3}}{8}$

We follow our previous notation $\Lambda_{3}=4$. In this case there is a $(3,1)$ superconformal field theory at $u=\frac{1}{2}$ where a triple singularity collides with a dyon singularity, and there is also a dyon point at $u=-\frac{19}{16}$. The discriminant is

$$
\Delta \sim\left(u-\frac{1}{2}\right)^{4}\left(u+\frac{19}{16}\right) .
$$

One can solve an algebraic equation and obtain an expression of $u$ in terms of gauge coupling $\tau$. Here we will use the normalization $\tau=\frac{i}{2 \pi} \frac{\partial^{2} F^{(0)}}{\partial a^{2}}$, which is one half of the T-dual of the gauge coupling we use in $N_{f}=3$ massless case previously in section 2 . There are two branches of solutions [42], and we take the branch where in the weak coupling limit $\tau \rightarrow i \infty$, the modulus goes like $u \rightarrow \infty$. The expression for $u$ is [42]

$$
u=\frac{27 E_{6} E_{4}^{\frac{3}{2}}+23 E_{4}^{3}+4 E_{6}^{2}}{8\left(E_{6}^{2}-E_{4}^{3}\right)}
$$

and there are also formulae for the derivative of period $a$

$$
\begin{aligned}
& \frac{d a}{d u}=\left(\frac{2}{27}\right)^{\frac{1}{2}}\left(\frac{E_{6}-E_{4}^{\frac{3}{2}}}{E_{4}}\right)^{\frac{1}{2}} \\
& \frac{d a}{d \tau}=-\left(\frac{27}{128}\right)^{\frac{1}{2}} \frac{E_{6}+E_{4}^{\frac{3}{2}}}{\left(E_{6}-E_{4}^{\frac{3}{2}}\right)^{\frac{1}{2}}} .
\end{aligned}
$$

We have not found an explicit formula for period $a(\tau)$. However, to write an exact formula for the topological string amplitude of the $F^{(g)}$ in terms of modular forms, we only need 
the derivative of period $a$. The genus one amplitude is

$$
\begin{aligned}
F^{(1)} & =-\frac{1}{2} \log \left(\frac{d a}{d u}\right)-\frac{1}{12} \log (\Delta) \\
& =-\frac{1}{12} \log \left(\frac{E_{4}^{3}\left(E_{6}+E_{4}^{\frac{3}{2}}\right)}{\left(E_{6}-E_{4}^{\frac{3}{2}}\right)^{2}}\right)
\end{aligned}
$$

where as usual we are not careful about an ambiguous additive constant in $F^{(1)}$.

As in the $N_{f}=1,2$ case, we can solve the flat coordinate $a$ and express the prepotential $F^{(0)}$ and genus one free energy $F^{(1)}$ in terms of it. Denote $z=u-\frac{1}{2}$, we found the solutions of Picard-Fuchs equation around $z=0$ as the followings

$$
\begin{aligned}
a & =z^{\frac{2}{3}}\left(1-\frac{8}{405} z+\frac{49}{13122} z^{2}-\frac{18928}{17537553} z^{3}+\mathcal{O}\left(z^{4}\right)\right), \\
\frac{\partial F^{(0)}}{\partial a} & =z^{\frac{4}{3}}\left(1-\frac{80}{567} z+\frac{242}{6561} z^{2}-\frac{254320}{20726199} z^{3}+\mathcal{O}\left(z^{4}\right)\right) .
\end{aligned}
$$

The genus zero and one free energy up to a constant are

$$
\begin{aligned}
& F^{(0)}=a^{3}-\frac{64}{945} a^{\frac{9}{2}}+\frac{401}{36450} a^{6}-\frac{885232}{351833625} a^{\frac{15}{2}}+\mathcal{O}\left(a^{9}\right) \\
& F^{(1)}=-\frac{1}{4} \log (a)-\frac{4}{135} a^{\frac{3}{2}}+\frac{3403}{437400} a^{3}-\frac{225869}{81192375} a^{\frac{9}{2}}+\mathcal{O}\left(a^{6}\right) .
\end{aligned}
$$

\section{The non-compact limit of Calabi-Yau compactifications}

A good way to solve the holomorphic anomaly equations for a Riemann surface of genus one describing an $N=2$ gauge theory is in terms of rings of anholomorphic modular forms, as we have seen in sections 2 and 3 .

However even without knowing anything about the structure of the modular forms w.r.t. the modular group of the family of curves, one can derive the necessary almost holomorphic objects directly in terms of the periods, which are solutions of the Picard-Fuchs equations. This has been done for compact Calabi-Yau spaces using special Kähler geometry [21] and the anholomorphic objects are the BCOV propagators. The derivatives that appear in the holomorphic anomaly equation close within a finitely generated polynomial ring of almost holomorphic modular functions and the $F^{(g)}$ are themselves such polynomials [25].

As explained in [6,9] extracting $4 \mathrm{~d} N=2$ non-perturbative gauge theory from type II string theory compactified on a Calabi-Yau space $W$ can be done geometrically by taking a limit in the geometrical parameter of the Calabi-Yau space in which part of the geometry decompactifies. Since we are dealing with the vector moduli space the limit is taken in the A-model in the complexified Kähler space and in the B-model on the mirror manifold the limit is taken in the complex structure space. For Calabi-Yau manifolds $W$ embedded in toric ambient spaces a wide class of limiting configurations in the B-model has been 
described as the 3 -d total space of a conic bundle over $\mathbb{C}^{*} \times \mathbb{C}^{*}$ branched at a Riemann surface $\mathcal{C}_{g}^{*} \in \mathbb{C}^{*} \times \mathbb{C}^{*}[6]$. For the relevant geometries the compactification of $\mathcal{C}_{g}^{*}$ is then the Seiberg-Witten curve $\mathcal{C}_{g}$. A general feature of the limit is that the periods of the $(3,0)$ form $\Omega$ over 3 -cycles in $W$, which stay finite become the periods of a meromorphic form $\lambda$ over 1 -cycles on $\mathcal{C}_{g}^{*}[9]$. The form $\lambda$ on $\mathcal{C}_{g}^{*}$ can be obtained by integrating $\Omega$ over the non-compact directions in the limiting configuration of $W$ [10].

Below we review in section 5.1 the important properties of the complex moduli space of complex Calabi-Yau manifolds following [26] and compare then the ring structure used to solve the holomorphic anomaly equation in the big phase space [26] with the one developed for the small phase space $[25,28,29]$.

Further we describe in the sections 5.2, 5.3 how the ring structure behaves in the noncompact limit extending the formalism of $[19,46]$ from local toric Calabi-Yau manifolds to non-conformal local gauge theory limits with matter fields. .

\subsection{Special geometry and rigid special geometry}

The origin of the anholomorphicity comes from the metric on the moduli space of the $N=2$ vector multiplets, which determines their kinetic term in the effective action. The latter is an $N=2$ supergravity action for the compact case and an $N=2$ super symmetric (gauge theory) action without gravity in the non-compact case. The vector multiplet moduli spaces are identified with the complex structure moduli space $\mathcal{M}$ of the Calabi-Yau $W$ and the Riemann-surface $\mathcal{C}_{g}^{*}$ respectively. The metrics are the Weil-Petersen metrics on these geometric moduli spaces. In both cases they derive from a real Kähler potential $K$ as $G_{i \bar{\jmath}}=\partial_{i} \bar{\partial}_{\bar{\jmath}} K$, but there is additional structure. For the Calabi-Yau case this is usually formulated as special Kähler geometry in the small phase space, i.e. in the inhomogeneous coordinates discussed below, and for the Riemann-surface the structure is always rigid special geometry. However in the homogeneous coordinates, also called the big moduli space, the $N=2$ supergravity action for the compact case can also be formulated in terms of rigid special geometry, which simplifies the limit to the local case.

The splitting of the middle de Rham cohomology of the compact Calabi-Yau $W$

$$
\begin{aligned}
& H^{3}(W, \mathbb{Z})= H^{3,0} \oplus H^{2,1} \oplus H^{1,2} \oplus H^{0,3} \\
& \Omega \quad \chi_{i} \quad \bar{\chi}_{\bar{\imath}} \quad \bar{\Omega}, \quad i, \bar{\imath}=1, \ldots, h^{21}
\end{aligned}
$$

into Hodge cohomology groups depends on the choice of complex structure. In particular we indicated the basis $\Omega, \chi_{i}, \bar{\chi}_{\bar{\imath}}, \bar{\Omega}, i, \bar{\imath}=1, \ldots, h^{21}$ of the individual Hodge cohomology groups that changes with the complex structure. One introduces a fixed topological and symplectic basis $\left(A^{K}, B_{K}\right)$ of $H_{3}(W, \mathbb{Z})$ and a dual symplectic basis $\left(\alpha_{K}, \beta^{K}\right)$ of $H^{3}(W, \mathbb{Z})$. Here $K=0, \ldots, h_{21}$ and the non vanishing pairings are $A^{L} \cap B_{K}=\int_{W} \alpha_{K} \wedge \beta^{L}:=$ $\left\langle\alpha_{K}, \beta^{L}\right\rangle=-\left\langle\beta^{L}, \alpha_{K}\right\rangle=\int_{A^{L}} \alpha_{K}=\int_{B_{K}} \beta^{L}=\delta_{K}^{L}$. If one expand $\Omega=X^{I} \alpha_{I}-F_{I} \beta^{I}$ and $\chi_{i}=\chi_{i}^{I} \alpha_{I}-\chi_{I i} \beta^{I}$ in terms of periods

$$
X^{I}=\int_{A^{I}} \Omega, \quad F_{i}=\int_{B_{I}} \Omega, \quad \chi_{i}^{I}=\int_{A^{I}} \chi_{i}, \quad \chi_{I i}=\int_{B_{I}} \chi_{i},
$$

the $X^{I}$ become homogeneous coordinates of the moduli space of complex structures. The dual periods $F_{I}=\int_{B_{I}} \Omega$ and the $\chi_{i}^{I}, \chi_{I i}$ are not independent but related to $X^{I}$ by special 
geometry. It is convenient to define $Y^{I}:=\lambda^{-1} X^{I}, I=0, \ldots, h_{21}$ and $\partial_{I}=\partial_{Y_{I}}$. It is easy to see that $\partial_{I_{1}}, \ldots, \partial_{I_{k}} \Omega \in \bigoplus_{l=0, k} H^{3-l, l}$. Transversality, i.e. $\langle a, b\rangle:=\int_{W} a \wedge b=0$ unless both Hodge indices $(p, q)$ of $a$ and $b$ add up to 3 , means that $\left\langle\Omega, \partial_{I} \Omega\right\rangle=\left\langle\Omega, \partial_{I} \partial_{J} \Omega\right\rangle=0$ and that implies the existence of a holomorphic prepotential $F^{(0)}(\underline{Y})=\frac{1}{2} \lambda^{2} Y^{I} F_{I}$. The latter is a homogeneous function of degree two in $Y^{I}$, such that $F_{I}=\frac{\partial F^{(0)}}{\partial Y^{I}}$. In the big moduli space, parameterized by the homogeneous coordinates $Y^{I}$, one defines a Kähler potential

$$
\mathcal{K}=\frac{i}{2}\left(Y^{K} \bar{F}_{K}-\bar{Y}^{K} F_{K}\right), \quad \mathcal{G}_{I J}=\partial_{I} \partial_{J} \mathcal{K}=\operatorname{Im} \tau_{I J}
$$

a symmetric weight zero tensor $\tau_{I J}=\frac{\partial^{2} F}{\partial Y_{I} \partial Y_{J}}$ and a symmetric weight -1 triple coupling $C_{I J K}=\partial_{I} \partial_{J} \partial_{K} F=\left\langle\Omega, \partial_{I} \partial_{J} \partial_{K} \Omega\right\rangle$. The metric $\mathcal{G}_{I J}$ has signature $\left(1, h_{21}\right)$. The connection is $\Gamma_{I J}^{K}=\mathcal{G}^{K \bar{L}} \partial_{J} \mathcal{G}_{I \bar{L}}=-\frac{i}{2} C_{J K}^{I}$ and one has the so called special geometry relation

$$
\left[D_{\bar{I}}, D_{J}\right]_{K}^{L}=\bar{\partial}_{\bar{I}} \Gamma_{J K}^{L}=\frac{1}{4} C_{J K P} \bar{C}_{\bar{I}}^{P L},
$$

which can be viewed as integrability condition for the existence of the holomorphic prepotential $F$, such that $C_{I J K}$ and $\mathcal{G}_{I \bar{J}}$ can be obtained from it by differentiation. It was shown in [26] that the holomorphic anomaly equation of [21] reads in the big moduli space

$$
\bar{\partial}_{\bar{I}} F^{(g)}=-\frac{i}{8} \bar{C}_{\bar{I}}^{J K}\left(D_{J} \partial_{K} F^{(g-1)}+\sum_{h=1}^{g-1} \partial_{J} F^{(h)} \partial_{K} F^{(g-h)}\right) .
$$

Since $\bar{D}_{\bar{I}} \bar{C}_{\bar{J} \bar{K} \bar{L}}=\bar{D}_{\bar{J}} \bar{C}_{\bar{I} \bar{J} \bar{J}}$ and $\bar{D}_{\bar{K}} \mathcal{G}_{I \bar{L}}=0$ one can integrate

$$
\partial_{\bar{K}} S^{I J}=\frac{i}{4} \bar{C}_{\bar{K}}^{I J}
$$

The $S^{I J}$ are anholomorphic tensors, called the propagators, ${ }^{13}$ they play a similar rôle that $\hat{E}_{2}$ plays for the elliptic curve. From (5.4) one can solve

$$
S^{K L}=i\left(C_{I}^{-1}\right)^{K P}\left(\Gamma_{I}\right)_{P}^{L}+\mathcal{H}^{K L} .
$$

Here $I$ is not summed over and $\mathcal{H}^{K L}$ is a holomorphic ambiguity. The latter must be chosen so that $S^{K L}$ is a tensor. The precise choice affects the form of the total ambiguity $f^{(g)}$, but is otherwise arbitrary. One convenient choice is to require that $\partial_{I} F^{(1)}=-\frac{i}{8} C_{I K L} S^{K L}$.

The idea of direct integration is based on the fact that all anholomorphic dependence of the modular invariant scalar $F^{(g)}$ is in the $S^{I J}$. Therefore by (5.6) $\bar{\partial}_{\bar{I}} F^{(g)}=\frac{i}{4} \bar{C}_{\bar{I}}^{J K} \frac{\partial F}{\partial S^{J K}}$ and $\bar{C}_{\bar{I}}^{J K}$ cancels from equation (5.5), which can then be directly integrated w.r.t. to $S^{J K}$ up to holomorphic terms $f^{(g)}$, which must also be modular invariant. To proceed in the iteration in $g$ one must show that the anholomorphic generators $S^{I J}$ close under the covariant derivative up to holomorphic terms. By considering $\bar{\partial}_{\bar{I}} D_{I} S^{J K}$, using (5.4) and integrating w.r.t $\bar{Y}^{\bar{I}}$ one gets

$$
D_{I} S^{J K}=C_{I M N} S^{N K} S^{M J}+\mathcal{H}_{I}^{J K}
$$

\footnotetext{
${ }^{13}$ Indeed the $F^{(g)}$ can be reconstructed with the propagators and vertices $\partial_{I_{1}}, \ldots, \partial_{I_{n}} F^{(h<g)}$ by Feynman rules of an auxiliary field theory [21] for the small phase space. For the formalism in the big phase space see $[26]$.
} 
Let us now come to special geometry in the small phase space, whose coordinates are the inhomogeneous variables $t^{i}=\frac{X^{i}}{X^{0}}, i=1, \ldots, h_{21}$. The Kähler potential $K$ in the small phase space is given by ${ }^{14}$

$$
e^{-K}=i \int_{W} \Omega \wedge \bar{\Omega}=i\left(X^{I} \bar{F}_{\bar{I}}-\bar{X}^{\bar{I}} F_{I}\right)=i\left(t^{i}-\bar{t}^{\bar{\imath}}\right)\left(\partial_{i} \mathcal{F}^{(0)}+\bar{\partial}_{\bar{\imath}} \overline{\mathcal{F}}^{(0)}\right)-2 i\left(\mathcal{F}^{(0)}-\overline{\mathcal{F}}^{(0)}\right) .
$$

Here we define $\left(X^{0}\right)^{2} \mathcal{F}^{(0)}(\underline{t})=F^{(0)}(\underline{X})$ using the degree 2 homogeneity of $F^{(0)}$ and the third equality holds up to a Kähler transformation.

The connection $\Gamma_{J K}^{I}$ splits into a metric connection, w.r.t. $G_{i \bar{\jmath}}=\partial_{i} \partial_{\bar{\jmath}} K$, and a Kähler connection. The covariant derivative becomes $D_{i}=\partial_{j}-\Gamma_{i}-k K_{i}$ for objects in $\mathcal{L}^{k} \otimes T^{*} \mathcal{M}$, with an analogous definition for $D_{\bar{\imath}}$. Holomorphic sections $A(\underline{t})$ of $\mathcal{L}^{k}$ transform like $A(\underline{t}) \rightarrow$ $A(\underline{t}) e^{-k h(\underline{t})}$ under Kähler transformations $K(\underline{t}, \underline{\bar{t}}) \rightarrow K(\underline{t}, \underline{\bar{t}})+h(\underline{t})+\bar{h}(\underline{\bar{t}})$. In particular the holomorphic $(3,0)$ form $\Omega \in \mathcal{L}$ and $F^{(g)} \in \mathcal{L}^{2-2 g}$. The covariant derivative eliminates the $(3,0)$ part in the derivative of $\Omega$ and hence $\chi_{i}=D_{i} \Omega\left(\bar{\chi}_{\bar{\imath}}=D_{\bar{\imath}} \bar{\Omega}\right)$. Applying this under the integral yields $\chi_{i}^{I}=D_{i} X^{I}$ and $\chi_{I i}=D_{i} F_{I}$, which serve as projectors from the big to the small phase space. In particular the triple coupling in inhomogeneous variables $C_{i j k} \in \mathcal{L}^{2} \otimes \operatorname{Sym}^{3} T^{*} \mathcal{M}$ are

$$
C_{i j k}=\left\langle\Omega, \partial_{i} \partial_{j} \partial_{k} \Omega\right\rangle=\sum_{I=0}^{h_{21}}\left(X^{I} \partial_{i} \partial_{j} \partial_{k} F_{I}-F_{I} \partial_{i} \partial_{j} \partial_{k} X^{I}\right)=\chi_{i}^{I} \chi_{j}^{J} \chi_{k}^{K} C_{I J K}
$$

It follows that $C_{i j k}=D_{i} D_{j} D_{k} \mathcal{F}^{(0)}(\underline{t})$. Using $\left\langle\chi_{i}, \bar{\chi}_{\bar{\imath}}\right\rangle=e^{-K} G_{i \bar{\imath}}$ from (5.9) and transversality one gets with the definition (5.10)

$$
D_{i} X^{I}=: \chi_{i}^{I}, \quad D_{i} \chi_{j}^{I}=i C_{i j k} G^{k \bar{k}} \bar{\chi}_{\bar{\jmath}}^{I} e^{K}, \quad D_{i} \bar{\chi}_{\bar{\imath}}^{I}=G_{i \bar{\imath}} \bar{X}^{I} .
$$

With $\left[D_{i}, \bar{D}_{\bar{\imath}}\right] \chi_{k}=-G_{i \bar{\imath}} \chi_{k}+R_{i \bar{\imath} k}^{l} \chi_{l}$ one arrives at the special Kähler relation in inhomogeneous coordinates

$$
\left[D_{i}, D_{\bar{\imath}}\right]_{j}^{k}=R_{i \bar{\imath} j}{ }^{k}=\bar{\partial}_{\bar{\imath}} \Gamma_{i j}^{k}=\delta_{i}^{k} G_{j \bar{\imath}}+\delta_{j}^{k} G_{i \bar{\imath}}-C_{i j l} C_{\bar{\imath}}^{k l} .
$$

The projection of the $S^{I J}$ is straightforward

$$
S^{I J}=\left(\begin{array}{ll}
X^{I} & \chi_{i}^{I}
\end{array}\right)\left(\begin{array}{cc}
S & -S^{i} \\
-S^{i} & S^{i j}
\end{array}\right)\left(\begin{array}{c}
X^{J} \\
\chi_{j}^{J}
\end{array}\right) .
$$

Here the relations $\bar{D}_{\bar{\imath}} \bar{C}_{\bar{\jmath} k \bar{l}}=\bar{D}_{\bar{\jmath}} \bar{C}_{\bar{\imath} \bar{l} \bar{l}}$ are integrated to $\bar{C}_{\bar{\imath}}^{j k}=\bar{\partial}_{\bar{\imath}} S^{j k}, G_{\bar{\imath} k} S^{k j}=\bar{\partial}_{\bar{\imath}} S^{j}$ and $G_{\bar{\imath} j} S^{j}=\frac{1}{2} \bar{\partial}_{\bar{\imath}} S$. The potentials $S^{i j}, S^{j}, S$, also called the propagators, allow to solve the anomaly equation, by partial integration, see for details [21], up to an holomorphic ambiguity. One can project the propagators from the big phase space or rederive them from the projected special Kähler relation (5.12). e.g. $S^{i j}$ is solved from (5.12)

$$
\Gamma_{i j}^{k}=\delta_{i}^{k} \partial_{j} K+\delta_{j}^{k} \partial_{i} K-C_{i j l} S^{k l}+\tilde{h}_{i j}^{k} .
$$

\footnotetext{
${ }^{14}$ We follow the conventions of [26].
} 
The analogs of the statement about the closing of the propagators (5.8) under $D_{i}$ are $[25,28]$

$$
\begin{aligned}
D_{i} S^{k l} & =\delta_{i}^{k} S^{l}+\delta_{i}^{l} S^{k}-C_{i n m} S^{k m} S^{l n}+h_{i}^{k l}, \\
D_{i} S^{j} & =2 \delta_{i}^{j}-C_{i k l} S^{k l}+h_{i}^{j k} K_{k}+h_{i}^{j}, \\
D_{i} S & =C_{i k l} S^{k} S^{l}+\frac{1}{2} h_{i}^{k l} K_{k} K_{l}+h_{i}^{l} K_{l}+h_{i}, \\
D_{i} K_{j} & =-K_{i} K_{j}-C_{i j k} S^{k}+C_{i j k} S^{k l} K_{l}+h_{i j}
\end{aligned}
$$

and are derived from special geometry similarly as (5.8). e.g. from $\bar{\partial}_{\bar{k}}\left(D_{i} S^{k l}\right)=\bar{\partial}_{\bar{k}}\left(\delta_{i}^{k} S^{l}+\right.$ $\left.\delta_{i}^{l} S^{k}-C_{i n m} S^{k m} S^{l n}\right)$ follows the first of the closing relations (5.15), etc.

One finds from the properties under Kähler transformations [21, 25, 28] that $\tilde{S}^{i j}=$ $S^{i j}, \tilde{S}^{j}=S^{i}-S^{i j} K_{j}$ and $\tilde{S}=S-S^{i} K_{i}+\frac{1}{2} S^{i j} K_{i} K_{i}$ are a complete set of anholomorphic generators of a polynomial ring that contains the $F^{(g)}$ as polynomials with holomorphic coefficients. Indeed one can write the holomorphic anomaly equation as

$$
\frac{\partial \mathcal{F}^{(g)}}{\partial S^{i j}}=\frac{1}{2}\left(D_{i} D_{j} \mathcal{F}^{(g-1)}+\sum_{h=1}^{g-1} D_{i} \mathcal{F}^{(g-h)} D_{j} \mathcal{F}^{(h)}\right)
$$

and integrate it up to holomorphic terms as a polynomial. Note that the derivatives of $\mathcal{F}^{(g)}$ w.r.t. $S^{j}, S$ and $K_{i}$, which naively occur at the left hand side, cancel. This cancellation is equivalent to the statement that the dependence of $F^{(g)}$ is through the combinations $\tilde{S}^{i j}, \tilde{S}^{j}$ and $\tilde{S}$.

\subsection{The non-compact limit}

Non-compact Calabi-Yau manifolds are mirror to

$$
u v=H(x, y ; \underline{z})
$$

where $u, v \in \mathbb{Z}, x, y \in \mathbb{C}^{*}$ and $\underline{z}$ are moduli of the geometry. The geometry is that of conic bundle, which branches over the locus

$$
H(x, y ; \underline{z})=0,
$$

which is a family Riemann surfaces $\mathcal{C}_{g}^{*}$ of genus $g$. Let $\lambda=\log (x) \frac{\mathrm{d} y}{y}$ be meromorphic differential and $\left(a^{i}, b_{i}\right)$ a symplectic basis of $H^{1}\left(\Sigma_{g}, \mathbb{Z}\right)$ then the rigid effective action has a Kähler potential ${ }^{15}$

$$
K=\frac{i}{2}\left(t^{i} \overline{\mathcal{F}}_{\bar{\imath}}-\bar{t}^{\overline{1}} \mathcal{F}_{i}\right)
$$

where $t^{i}=\int_{a^{i}} \lambda$ and $\mathcal{F}_{i}=\int_{b_{i}} \lambda$. Note that the form of $K$ is like in (5.3), but the $t^{i}$ are directly appropriate flat local coordinates. The metric reads

$$
G_{i \bar{\jmath}}=\partial_{i} \bar{\partial}_{\bar{\jmath}} K=\frac{1}{2 i}\left(\tau_{i j}-\bar{\tau}_{\bar{\imath} \bar{\jmath}}\right)
$$

where $\tau_{i j}=\frac{\partial^{2} \mathcal{F}}{\partial t^{i} \partial t^{j}}$.

\footnotetext{
${ }^{15}$ We use here conventions, which differ by a factor $i$ multiplying the prepotential from the ones used in $(2.15)$ and call the flat coordinates $t^{i}, \mathcal{F}_{i}$ instead of $a^{i}, a_{D_{i}}$.
} 
In the local case one has the following simplifications. The Kähler connection in $D_{i}$ becomes trivial, and the $S^{l}$ as well as the $S$ (see [47]) vanish, i.e. the first equation in (5.15) and the equation (5.8) become equivalent and read

$$
D_{i} S^{k l}=-C_{i n m} S^{k m} S^{l n}+f_{i}^{k l} .
$$

The $S^{i j}$ are the generators of the ring of anholomorphic objects Since the Kähler connection $\partial_{j} K$ in (5.14) drops out, so the $S^{j i}$ are solved from

$$
\Gamma_{i j}^{k}=-C_{i j l} S^{k l}+\tilde{f}_{i j}^{k}
$$

as

$$
S^{i j}=-\left(C_{p}\right)^{i l}\left[\left(\Gamma_{p}\right)_{l}^{j}+\left(\tilde{f}_{p}\right)_{l}^{j}\right], \quad \forall p=1, \ldots, r .
$$

Here $r$ is the number of Kähler parameter in the mirror to 5.17. It has been pointed out e.g. in [19] that there are in general algebraic relations between the $S^{i j}$. If $\mathcal{C}_{f}$ has genus $g=1$ there will be only one independent $S^{i j}$, for $g=2$ there should be 3 independent $S^{i j}$. Again $p$ is not summed over in (5.23) and this over determined system requires a suitable choice of the ambiguity $\tilde{f}_{i j}^{k}$. This choice is simplified by the fact [48] that $\mathrm{d}_{i} F_{1}$ can be expressed through the propagator as

$$
\partial_{i} F_{1}=\frac{1}{2} C_{i j k} S^{j k}+A_{i}
$$

with an ambiguity $A_{i}$, which can be determined by the ansatz $A_{i}=\mathrm{d}_{i}\left(a \log \Delta+b_{j} \log z_{j}\right)$. Moreover the universal behavior of $F_{1}$ near the conifold locus [36] implies $a=-\frac{1}{12}$.

The above construction of the an-holomorphic modular objects applies to the noncompact geometries that relates the Calabi-Yau rings of [21, 25, 26, 28, 29] to the classical rings of almost holomorphic modular forms of subgroups of $\mathrm{SL}(2, \mathbb{Z})$ for $\mathrm{SU}(2)$ gauge groups [7] or $S P(2 g, \mathbb{Z})$ for $\mathrm{SU}(g+1, \mathbb{Z})$ gauge theories groups ${ }^{16}[1,3]$. Therefore it is possible to write the generators of the anholomorphic rings that are needed to solve the holomorphic anomaly equation for gauge theories in terms of solutions of the Picard-Fuchs equations. For example for $\mathrm{SU}(3)$ in terms of the solutions for the Appel differential system [38].

\subsection{Monodromy action}

The monodromy acts for the compact Calabi-Yau manifold $W$ as a subgroup of $S P\left(h_{3}(W), \mathbb{Z}\right)$ on the CY periods $\left(F_{I}=\partial_{I} F, X^{I}\right)^{T}$, i.e. as

$$
\left(\begin{array}{c}
\tilde{F}_{I} \\
\tilde{X}^{I}
\end{array}\right)=\left(\begin{array}{ll}
A_{I}^{J} & B_{I J} \\
C^{I J} & D^{I}{ }_{J}
\end{array}\right)\left(\begin{array}{c}
F_{J} \\
X^{J}
\end{array}\right)
$$

\footnotetext{
${ }^{16}$ Seiberg-Witten curves are by now known for any gauge group. In general they are special families of complex curves, whose deformation parameters correspond to vev of fields in the Cartan subalgebra of the group, see [45] for a review. However we expect the holomorphic anomaly and the gap condition discussed here only in the non-conformal cases.
} 
with all entries of $A_{I}^{J}, B_{I J}, C^{I J}$ and $D_{J}^{I}$ integers and

$$
\left(\begin{array}{ll}
A_{K}^{S} & \left(C^{T}\right)^{S K} \\
\left(B^{T}\right)_{S K} & D_{T}^{K}
\end{array}\right)\left(\begin{array}{cc}
0 & -\delta^{K}{ }_{I} \\
\delta_{K}^{I} & 0
\end{array}\right)\left(\begin{array}{ll}
A_{I}{ }^{P} & B_{I P} \\
C^{I P} & D^{I}{ }_{P}
\end{array}\right)=\left(\begin{array}{cc}
0 & -\delta^{S}{ }_{P} \\
\delta_{S}{ }^{P} & 0
\end{array}\right) .
$$

One clear advantage of the big phase space is that the monodromy acts simply on the tensors in the homogeneous coordinates. e.g. $\tau$ transforms as

$$
\tilde{\tau}_{I J}=(A \tau+B)_{I K}(C \tau+D)^{-1} K_{J}
$$

and modular objects of tensor weight $-N$ transform like $\tilde{C}_{I_{1}, \ldots, I_{n}}=(C \tau+$ $D)_{I_{1}}^{-1 K_{1}}, \ldots,(C \tau+D)_{I_{N}}^{-1 K_{N}} C_{K_{1}, \ldots, K_{N}}$.

The monodromy for the non-compact cases acts on the periods $\Pi^{T}=\left(\mathcal{F}_{i}=\int_{b_{i}} \lambda, t^{i}=\right.$ $\left.\int_{a^{i}} \lambda, m_{\mu}=\int_{\gamma_{\mu}} \lambda\right)$, where $a^{i}, b_{i}$ is a symplectic basis of $H_{1}\left(\mathcal{C}_{g}, \mathbb{Z}\right)$ and $\gamma_{\mu}$ are cycles encircling the points where $\lambda$ has a pole with non-vanishing residua. As mentioned above $\Pi^{T}$ can be obtained as the periods of $W$ which stay finite in the non-compact limit. ${ }^{17}$ We call $\mathcal{C}_{g}^{*}=\mathcal{C}_{g} \backslash\left\{p_{i}\right\}$. The monodromy acting on $H_{1}\left(\mathcal{C}_{g}, \mathbb{Z}\right)$ is a subgroup of $\operatorname{SP}(2 g, \mathbb{Z})$. The action on $\Pi$ is

$$
\left(\begin{array}{c}
\tilde{\mathcal{F}}_{i} \\
\tilde{t}^{i} \\
m_{\mu}
\end{array}\right)=\left(\begin{array}{ccc}
a_{i}^{j} & b_{i j} & l_{i \mu} \\
c^{i j} & d^{i} & l_{j}^{i} \\
0 & 0 & \mathbb{I}
\end{array}\right)\left(\begin{array}{c}
\mathcal{F}_{j} \\
t^{j} \\
m_{\mu}
\end{array}\right)
$$

and analogous to (5.26) we have from the preservation of the intersection form $a^{T} c=c^{t} a$, $b^{T} d=d^{T} b$ and $a^{T} d-c^{T} b=\mathbb{I}$, with all entries of $a, b, c, d$ and $l_{i \mu}, l_{\mu}^{i}$ integer. If $\mathcal{C}_{g}^{*}$ is obtained by a non-compact limit from $W$ the monodromy group of $\mathcal{C}_{g}^{*}$ generated by 5.28 is a subgroup of the monodromy group of $W$. The action on $\tau_{i j}$ is given similarly as in (5.27) by

$$
\tilde{\tau}_{i j}=(a \tau+b)_{i k}(c \tau+d)^{-1}{ }_{j} .
$$

An important difference is in the properties of the matrix $\tau$. In the global case $\operatorname{Im}\left(\tau_{I J}\right)$ has signature $\left(1, h_{21}\right)$, i.e. one negative eigenvalue. On the other hand as it was mentioned in section $(2.1)$ it is a key property of the solution of $[7,8,38]$ that $\operatorname{Im}\left(\tau_{i j}\right), i=1, \ldots, \operatorname{rank}(G)$, with $\tau_{i j}=\frac{i}{2 \pi} \partial_{a^{i}} \partial_{a^{j}} F^{(0)}$ is positive definite. Mathematically $\tau_{i j}$ defines the Siegel upper half space associated to $\mathcal{C}_{g}$. In the non-compact limit the matrix $\operatorname{Im}\left(\tau_{I J}\right)$ is therefore projected a positive definite submatrix.

The Hodge star operator $*$ on $W$ defines a natural complex structure on $H^{3}(W)$, which is $+i$ on $H^{3,0} \oplus H^{1,2}$ and -1 on $H^{2,1} \oplus H^{0,3}$. This leads to the so called Weil intermediate Jacobian, which comes with a natural pairing given by the anholomorphic matrix

$$
N_{I J}=\tau_{I J}-2 i \frac{\operatorname{Im}\left(\tau_{I K}\right) \operatorname{Im}\left(\tau_{J L}\right) \bar{X}^{L} \bar{X}^{K}}{\operatorname{Im}\left(\tau_{K L}\right) \bar{X}^{L} \bar{X}^{K}} .
$$

It is well known in supergravity that this defines the matrix of theta angles and the gauge couplings as $N_{I J}=: \frac{\Theta_{I J}}{\pi}+8 \pi i\left(g^{-2}\right)_{I J}$ and that $\operatorname{Im}\left(N_{I J}\right)$ is positive. In the non-compact

\footnotetext{
${ }^{17}$ Typically the fundamental period $X^{0}$ in the large radius limit becomes one of the constant periods $m_{\mu}$ see e.g. the discussion of local $\mathcal{O}(-3) \rightarrow \mathbb{P}^{2}$ in the large fiber limit of the elliptic fibration over $\mathbb{P}^{2}$ realized as $X_{18}(1,1,1,6,9)$, see [19].
} 
limit certain a submatrix of the anholomorphic $N_{I J}$ becomes the holomorphic $\tau_{i j}$ of the rigid gauge theory. The Griffith complex structure on $H^{3}(W)$ is defined by $+i$ on $H^{3,0} \oplus H^{2,1}$ and -1 on $H^{1,2} \oplus H^{0,3}$ and the paring is given by $\tau_{I J}$, which as mentioned above has one negative eigenvalue. We note that $\tau_{I J}$ and $N_{I J}$ transform in the same way under $S P\left(h_{3}(W), \mathbb{R}\right)$ transformations.

\section{Matrix model approach}

The study of the gravitational couplings of SU(2) Seiberg-Witten theory has been a fruitful setting to explore various approaches for solving topological expansions. As reviewed in the introduction one can obtain the gravitational coupling $F^{(g)}$ by geometric engineering from toric Calabi-Yau 3-folds and by Nekrasov's instanton counting calculations. Both approaches, the former via the vertex formalism, lead to sums over partitions, which are valid in one region in the moduli space. In the geometric engineering approach one has in addition to take a limit. The direct integration of holomorphic anomaly equation [1] studied in the previous sections yields an analytic description of the higher genus amplitudes, which is recurse in the genus, but valid throughout the moduli space.

In this section we will turn to another approach, namely the matrix model method. The matrix model is in principle a framework that encodes exact perturbative information and possible non-perturbative completions. It was pioneered by Dijkgraaf and Vafa in particular in [13]. Following these suggestions the authors of [11] computed the gravitational couplings of $\mathcal{N}=2$ Seiberg-Witten theory by a limit from the Hermitian matrix model describing the glueball superpotential of $\mathcal{N}=1$ gauge theory. However, only the genus one amplitude $F^{(1)}$ has been obtained in this way, and it is not clear how to compute higher genus amplitudes in this approach, because $F^{(g)}$ is not gauge invariant for $g>1$ [11].

A microscopic matrix model was recently derived in [16] from the partition function $[4$, 5] using the matrix model descriptions of infinite partitions [15]. Motivated by the recent works of [20,49], we will apply the formalism in [20] to the topological expansion of SU(2) Seiberg-Witten theory. The formalism of [20] has been developed from the study of loop equations in matrix models. It also proceeds recursively genus by genus. One advantage of the formalism is that one no longer need to refer to a matrix model in this set up. The defining date are the spectral curve $\mathcal{C}$ and the differential $\lambda$, which yields the filling fraction and the open one point function. The Seiberg-Witten curve has been shown to be the spectral curve of the microscopic matrix model considered in [16]. It also follows from a double scaling limit of the spectral curve of Gross-Witten matrix model considered by Dijkgraaf and Vafa in [13].

One obvious advantage of the matrix model approach is that it gives also the open amplitudes. Given the local mirror curve $\mathcal{C}^{*}$ and the meromorphic differential $\lambda$ for topological string theory on local Calabi-Yau manifolds the matrix model predictions for the open amplitudes have been checked against the topological vertex results [18, 23]. The interpretation of these amplitudes in the gauge theory context is less clear. 


\subsection{Review of the formalism}

Here we review the formalism developed by Eynard and Orantin for integrating the loop equation. For more details and references see [20].

The algorithm is particularly elegant for elliptic curves in Weierstrass form. We will therefore focus on Seiberg-Witten curves in the Weierstrass form

$$
y^{2}=4 x^{3}-g_{2}(u) x-g_{3}(u) .
$$

Here is $u$ is the Coulomb modulus of the Seiberg-Witten theory. For the massless $N_{f}=2$ theory we find by transforming (2.2) into Weierstrassform

$$
g_{2}(u)=\frac{4}{3}\left(u^{2}+3\right), \quad g_{3}(u)=\frac{8 u}{27}\left(u^{2}-9\right),
$$

where the three roots of $(6.1)$ are $x=\frac{2 u}{3}, 1-\frac{u}{3},-1-\frac{u}{3}$ respectively. We note that is also the Weierstrass form for the Seiberg-Witten curve for pure $\mathrm{SU}(2)$ gauge theory as quoted in [7], while if we transform the $N_{f}=0$ case [30] in (2.2) into Weierstrass form we obtain

$$
g_{2}(\tilde{u})=-1+\frac{4 \tilde{u}^{2}}{3}, \quad g_{3}(u)=\frac{1}{27}\left(9 \tilde{u}-8 \tilde{u}^{3}\right) .
$$

The two curves specified by (6.1) with (6.2) or (6.3) respectively are known to be isogeneous. That means in particular that the Picard-Fuchs equation are the same, but a careful analysis of the integral basis of $H_{1}\left(\mathcal{C}_{1}, \mathbb{Z}\right)$ reveals the $b$ periods differ by a factor of two. The relation between $\tilde{u}$ and $u$ from the comparison of the $J$-function (2.7) is $u=\frac{\tilde{u}}{\sqrt{\tilde{u}^{2}-1}}$, i.e. it exchanges the asymptotic free region and the monopole region [38]. At genus zero it is difficult to distinguish the curves. Since the Picard-Fuchs equations are the same the holomorphic prepotential can be derived from any of them. However at genus one there is an important difference. We know that for $N_{f}=0$ the conifold factor is $\Delta \sim\left(u^{2}-1\right)$, while for the massless $N_{f}=2$ case it is $\Delta \sim\left(u^{2}-1\right)^{2}$, see (2.49) and (3.15). By calculating $\Delta$ from (2.8), we see that (6.1), (6.2) is the $N_{F}=2$ curve. Now an important simplification for the application of the [20] formalism arises if the meromorphic differential $\lambda$ is simple rational function of the Weierstrass $\mathcal{P}$-function for the curve written in the Weierstrass form. It turns out that for the Weierstrass curve of $N_{f}=2$ the form of the differential $\lambda=\frac{\sqrt{2}}{2 \pi} y \frac{d x}{x^{2}-1}$ used in [7] for the cubic curve quoted there as $N_{f}=0$ curve has this property, see (6.17). On the other hand if we transform the meromorphic (2.3) for $N_{f}=0$ to the Weierstrass representation we cannot express it as a rational function of the Weierstrass $\mathcal{P}$-function. As we mention above for genus zero prepotential it is not relevant to match precisely the correct pair of curve and differential, but for higher genus it is crucial. Below we stick to the technically simplest case namely the $N_{f}=2$ case.

Given a curve $\mathcal{C}_{g}$ the associated Bergmann kernel is defined as the unique bilinear meromorphic form with a single pole of degree 2 , whose integral over the $A$-cycles vanish, see [20] for details. For the family of genus one curves (6.1), the associated Bergmann kernel $B(p, q)$ is simply the Weierstrass $\mathcal{P}$-function plus a constant $X$

$$
B(p, q)=(\wp(p-q)+X) d p d q .
$$


The Weierstrass $\mathcal{P}$ function is a double periodic, even function on $\mathbb{C}$

$$
\wp\left(p+2 a_{1}\right)=\wp(p), \quad \wp\left(p+2 a_{2}\right)=\wp(p), \quad \wp(-p)=\wp(p),
$$

which has a double pole around the origin and the series expansion

$$
\wp(p)=\frac{1}{p^{2}}+\frac{g_{2}}{20} p^{2}+\frac{g_{3}}{28} p^{4}+\mathcal{O}\left(p^{6}\right) .
$$

In particular the $\mathcal{P}$-function is well defined on the two torus $\mathcal{C}_{1}=\mathbb{C} / \Lambda$, where $\Lambda$ is the lattice spanned by the periods $\left(2 a_{1}, 2 a_{2}\right)$. The complex structure of $\mathcal{C}_{1}$ is $\tau=\frac{a_{2}}{a_{1}}$ and $\left(a_{1}, a_{2}\right)$ are half periods.

The constant $X$ can be fixed by the A-cycle integral

$$
\int_{0}^{2 a_{1}} B(p, q)=\left(-2 \zeta\left(a_{1}\right)+2 a_{1} X\right) d p
$$

where $\zeta(p)$ is the Weierstrass zeta function, and its value at half period is related to the second Eisenstein series of $\tau$ as

$$
\zeta\left(a_{1}\right) a_{1}=\frac{\pi^{2}}{12} E_{2}(\tau) .
$$

Using the relations between Weierstrass invariants and the Eisenstein series

$$
\left(2 a_{1}\right)^{4} g_{2}(u)=\frac{4 \pi^{4}}{3} E_{4}(\tau), \quad\left(2 a_{1}\right)^{6} g_{3}(u)=\frac{8 \pi^{6}}{27} E_{6}(\tau)
$$

and the vanishing of (6.7), we determine $X$ in (6.4)

$$
X=\frac{3 g_{3}(u) E_{2}(\tau) E_{4}(\tau)}{2 g_{2}(u) E_{6}(\tau)} .
$$

Because of (2.20) the Bergmann Kernel transforms with a shift under modular transformations. One can define the modular invariant modified Bergmann kernel by replacing $E_{2}$ in (6.4) with $\hat{E}_{2}$, as defined in (2.19). This replacement induces an isomorphism between ring of quasimodular forms and the ring of almost holomorphic modular forms. In the manipulations below we can work with $E_{2}$ and replace it at the end of calculations with $\hat{E}_{2}$, if we wish to consider truly modular objects.

The Eisenstein series are related to Jacobi theta functions by the well-known formulae

$$
\begin{aligned}
& E_{4}(\tau)=b^{2}+b d+d^{2} \\
& E_{6}(\tau)=\frac{1}{2}\left(2 d^{3}+3 b d^{2}-3 b^{2} d-2 b^{3}\right),
\end{aligned}
$$

where $b, c, d$ are defined in (2.52). The modulus $u$ and half period $a_{1}$ can be written in terms of Jacobi theta function using (6.11) and (6.9) as ${ }^{18}$

$$
u=1+\frac{2 d}{b}, \quad a_{1}^{2}=\frac{\pi^{2} b}{8}
$$

\footnotetext{
${ }^{18}$ One can solve for $u$ by eliminating $a_{1}$ in (6.9). There are other solutions besides the solution $u=1+\frac{2 d}{b}$ we use. They correspond to various special points in the Coulomb moduli space as $\tau \rightarrow i \infty$, or are related the one we use by $Z_{2}$ symmetry. Without loss of generality we will just use the solution $u=1+\frac{2 d}{b}$ in order to compare with large $u$, i.e. weak coupling limit.
} 
Therefore the constant in (6.10) can be written in terms of modular forms

$$
X=\frac{2 E_{2}(\tau)}{3 b}
$$

In the Weierstrass form (6.1) the Seiberg-Witten curve is parameterized by the Weierstrass function and its derivative via the identification

$$
y=\wp^{\prime}(p), \quad x=\wp(p) .
$$

The branching points of the algebraic curve (6.1) are the points of $d x=0$, which are simply the half periods $a_{1}, a_{2}, a_{3}=a_{1}+a_{2}$ in the case of Weierstrass function. The values of Weierstrass function at half periods are the roots of Weierstrass equation $4 x^{3}-g_{2} x-g_{3}=0$. The ordering will not be important for us, so without loss of generality we can take

$$
\wp\left(a_{1}\right)=\frac{2 u}{3}, \wp\left(a_{2}\right)=1-\frac{u}{3}, \wp\left(a_{3}\right)=-1-\frac{u}{3} .
$$

The derivative of Weierstrass function vanishes at the half periods $\wp^{\prime}\left(a_{1}\right)=\wp^{\prime}\left(a_{2}\right)=$ $\wp^{\prime}\left(a_{3}\right)=0$. For a point $p$ near each branching point $a_{i}$, there is an unique image denoted as $\bar{p}$ such that $\wp(p)=\wp(\bar{p})$. Since the Weierstrass function satisfies $\wp\left(2 a_{i}-p\right)=\wp(p)$, we can easily determine

$$
\bar{p}=2 a_{i}-p
$$

Higher derivatives of Weierstrass function can be related to Weierstrass function and its derivative algebraically, for example we have the formula for the second derivative as $\wp^{\prime \prime}(p)=-\frac{g_{2}}{2}+6 \wp(p)^{2}$, etc.

The periods of Seiberg-Witten theory should correspond to the "filling fraction" defined in [20]. In the $N_{f}=2$ massless theory it is the integral of the following meromorphic differential

$$
\begin{aligned}
\lambda(p) & =\frac{1}{2 \sqrt{2}} \frac{y(p) d x(p)}{\left(x(p)-\wp\left(a_{2}\right)\right)\left(x(p)-\wp\left(a_{3}\right)\right)} \\
& =\frac{1}{2 \sqrt{2}} \frac{\wp^{\prime}(p)^{2} d p}{\left(\wp(p)-\wp\left(a_{2}\right)\right)\left(\wp(p)-\wp\left(a_{3}\right)\right)}
\end{aligned}
$$

over the cycles of algebraic curve, i.e. $a=\frac{1}{2 \pi i} \int_{a} \lambda(p)$. Here we have chosen a normalization for which the derivative of the prepotential is $\frac{\partial^{2} F^{(0)}}{\partial a^{2}}=-2 \pi i \tau$. This will be convenient later on.

A set of diagrammatic rules are provided in [20] to construct the topological expansion $F^{(g)}$ associated with the algebraic curve. Below we list the basic components and their expansions around the branching points $a_{i}, i=1,2,3$ :

1. The vertex $\omega(p)$. This can be constructed from the differential one-form in (6.17) as the following,

$$
\begin{aligned}
\omega(p) & =\frac{1}{2 \sqrt{2}} \frac{(y(p)-y(\bar{p})) d x(p)}{\left(x(p)-\wp\left(a_{2}\right)\right)\left(x(p)-\wp\left(a_{3}\right)\right)} \\
& =\frac{1}{\sqrt{2}} \frac{\wp^{\prime}(p)^{2} d p}{\left(\wp(p)-\wp\left(a_{2}\right)\right)\left(\wp(p)-\wp\left(a_{3}\right)\right)}
\end{aligned}
$$


It is straightforward to compute the series expansion near the branching points. For the branching point $a_{1}$, the vertex $\omega(p)$ goes like $\omega(p) \sim\left(p-a_{1}\right)^{2}$, while the other two points $a_{2}$ and $a_{3}$, the vertex goes like $\omega(p) \sim \mathcal{O}(1)$.

2. The root $\Phi(p)$. This is simply the integral of the differential one-form $\lambda(p)$ in (6.17) from any base point on the algebraic curve

$$
\Phi(p)=\int^{p} \lambda(p)
$$

The integration constant will not appear in final answers and will not be important.

It is straightforward to compute the series expansion of $\lambda(p)$ near the branching points and perform the integral. At the branching point $a_{1}$ the root $\Phi(p)$ has the leading behaviour $\Phi(p) \sim\left(p-a_{1}\right)^{3}$, while at the other two points $a_{2}$ and $a_{3}$, it behaves like $\Phi(p) \sim\left(p-a_{i}\right)$.

3. The line-propagator is simply the Bergmann kernel $B(q, p)$. We expand it in the first variable $q$ around a branching point $a_{i}$,

$$
\begin{aligned}
\frac{B(q, p)}{d p d q}= & \wp\left(a_{i}-p\right)+X+\wp^{\prime}\left(a_{i}-p\right)\left(q-a_{i}\right) \\
& +\frac{1}{2} \wp^{\prime \prime}\left(a_{i}-p\right)\left(q-a_{i}\right)^{2}+\mathcal{O}\left(\left(q-a_{i}\right)^{3}\right) .
\end{aligned}
$$

We then expand in the second variable $p$ around another branching point $a_{j}$. If $a_{i}=a_{j}$, there will be poles as $p \rightarrow a_{i}$. For $a_{i} \neq a_{j}$, there will be no pole. In both cases it is straightforward obtain the series expansions.

4. The arrow-propagator $d E_{q}(p)$ is an integral of the Bergmann kernel and can be expanded around a branching point $a_{i}$ in the following way

$$
\begin{aligned}
\frac{d E_{q}(p)}{d p}= & \frac{1}{2} \int_{q}^{\bar{q}} B(\xi, p) \\
= & -\left(\wp\left(a_{i}-p\right)+X\right)\left(q-a_{i}\right)-\frac{1}{6} \wp^{\prime \prime}\left(a_{i}-p\right)\left(q-a_{i}\right)^{3} \\
& -\frac{1}{120} \wp^{(4)}\left(a_{i}-p\right)\left(q-a_{i}\right)^{5}+\cdots
\end{aligned}
$$

Again, if $a_{i}=a_{j}$, there will be poles as $p \rightarrow a_{i}$, otherwise for $a_{i} \neq a_{j}$, there will be no pole. The necessary series expansions are straightforward to obtain.

From these basic components one can construct the correlation functions $W_{k}^{(g)}\left(p_{1}, \cdots, p_{k}\right)$, and free energy $F^{(g)}$ for all $g \geq 2$ in terms of some residue formulae. For example, The genus one one-point function is

$$
W_{1}^{(1)}(p)=\operatorname{Res}_{q \rightarrow \mathbf{a}} \frac{d E_{q}(p)}{\omega(q)} B(q, \bar{q})
$$


and the genus two free energy $F^{(2)}$ is

$$
\begin{aligned}
F^{(2)}= & -\frac{1}{2} \operatorname{Res}_{p \rightarrow \mathbf{a} \operatorname{Res} \operatorname{Res}_{q \rightarrow \mathbf{a}} \operatorname{Res}\left\{\frac{\Phi(p) d E_{q}(p)}{\omega(q)} \frac{d E_{r}(q)}{\omega(r)} \frac{d E_{s}(\bar{q})}{\omega(s)} B(r, \bar{r}) B(s, \bar{s})\right.} \\
& +\frac{\Phi(p) d E_{q}(p)}{\omega(q)} \frac{d E_{r}(q)}{\omega(r)} \frac{d E_{s}(\bar{r})}{\omega(s)} B(r, \bar{q}) B(s, \bar{s})+\frac{\Phi(p) d E_{q}(p)}{\omega(q)} \frac{d E_{r}(q)}{\omega(r)} \frac{d E_{s}(r)}{\omega(s)} \\
& \times[B(\bar{r}, \bar{q}) B(s, \bar{s})+B(\bar{s}, \bar{q}) B(s, \bar{r})+B(s, \bar{q}) B(\bar{s}, \bar{r})]\}
\end{aligned}
$$

where the residues are taken around the three branching points $a_{1}, a_{2}, a_{3}$.

\subsection{Calculations of open and close amplitudes}

We calculate the genus one one-point function $W_{1}^{(1)}(p)$ and the genus two free energy $F^{(2)}$ for the Seiberg-Witten curve (6.1). As we mentioned it describes the SU(2) Seiberg-Witten theory with two massless flavors. The genus one one-point function $W_{1}^{(1)}(p)$ is calculated from (6.22), we find

$$
\begin{aligned}
W_{1}^{(1)}(p)= & \sum_{i=1}^{3} \operatorname{Res}_{q \rightarrow a_{i}} \frac{d E_{q}(p)}{\omega(q)} B(q, \bar{q}) \\
= & -\frac{d p}{48 \sqrt{2}\left(u^{2}-1\right)}\left[4(u-6 X)\left(\wp\left(p-a_{1}\right)+X\right)+6(u-1)\left(\wp\left(p-a_{2}\right)+X\right)\right. \\
& \left.+6(u+1)\left(\wp\left(p-a_{3}\right)+X\right)-\wp^{\prime \prime}\left(p-a_{1}\right)\right] .
\end{aligned}
$$

The genus one free energy $F^{(1)}$ is not directly constructed from the diagrammatic rules, but the derivative of it with respect to the Seiberg-Witten period is the integral of $W_{1}^{(1)}(p)$ over the B-cycle

$$
\frac{\partial F^{(1)}}{\partial a}=\int_{0}^{2 a_{2}} W_{1}^{(1)}(p)
$$

Using the formulae for Weierstrass zeta function $2 a_{2} \zeta\left(a_{1}\right)-2 a_{1} \zeta\left(a_{2}\right)=\pi i$ and (6.12) we can compute the integral

$$
\frac{\partial F^{(1)}}{\partial a}=\int_{0}^{2 a_{2}} W_{1}^{(1)}(p)=\frac{i \sqrt{b}}{6 c d}\left(E_{2}-b-2 d\right) .
$$

This matches with our earlier calculations for Seiberg-Witten theory with $N_{f}=2$ massless flavors, using (2.50), (2.51). ${ }^{19}$

\footnotetext{
${ }^{19}$ There is an extra factor of $i$ comparing with (2.50), (2.51). This is because the matrix model should describe the expansion of $F^{(g)}$ around the conifold point for which the filling fraction is real and goes to zero, instead of the point $u \rightarrow \infty$. The formulae (6.12) we have used are for the point at infinity $u \rightarrow \infty$, and should become $u=1+\frac{2 b}{d}, a_{1}^{2}=-\frac{\pi^{2} d}{8}$ for the conifold point. The extra factor of $i$ is then cancelled due to the extra minus sign of $a_{1}^{2}$. Since this problem will not appear at higher genus $g \geq 2$, we will still use the convention at $u \rightarrow \infty$ for convenience in comparing with instanton counting.
} 
Now we come to genus two free energy, we compute the various terms in (6.23) and the total result is

$$
F^{(2)}=\frac{675 X^{3}-1350 u X^{2}+\left(990 u^{2}+1350\right) X-16 u^{3}-1080 u}{6480\left(u^{2}-1\right)^{2}} .
$$

Substituting in $u=1+\frac{2 d}{b}$ and $X=\frac{2 E_{2}}{3 b}$, we find the agreement with earlier calculations (2.54) for Seiberg-Witten theory with $N_{f}=2$ massless flavors using holomorphic anomaly.

Similarly, we can compute the genus two one-point function

$$
\begin{aligned}
W_{1}^{(2)}(p)= & \frac{5}{32 \sqrt{2}\left(u^{2}-1\right)^{3}}\left[X^{5}+\frac{6 u \wp(p)+3-7 u^{2}}{2 u-3 \wp(p)} X^{4}\right. \\
& +\frac{9\left(75+77 u^{2}\right) \wp(p)^{2}-12 u\left(75+77 u^{2}\right) \wp(p)+2\left(405-660 u^{2}+559 u^{4}\right)}{45(2 u-3 \wp(p))^{2}} X^{3} \\
& \left.+b_{2} X^{2}+b_{1} X+b_{0}\right]
\end{aligned}
$$

where $b_{0}, b_{1}, b_{2}$ are some very complicated functions of the Weierstrass function $\wp(p)$ and $u{ }^{20}$ Some empirical remarks can be made about a genus $g$ one-point amplitude ${ }^{21} W_{1}^{(g)}(p)$ :

1. $W_{1}^{(g)}(p)$ is a polynomial of $X$ of degree $3 g-1$.

2. The coefficients of the polynomial are rational functions of $\wp(p)$ and $u$. They are regular at $p=0$ (or equivalently $\wp(p)=\infty$ ). They are singular at the half periods $p=a_{1}, a_{2}, a_{3}$. The degree of poles of $\wp(p)-\wp\left(a_{1}\right), \wp(p)-\wp\left(a_{2}\right), \wp(p)-\wp\left(a_{3}\right)$ are $g+3,2,2$ respectively. For example, the coefficient $b_{0}$ in (6.28) as a rational function $\wp(p)$ can be written as

$$
\begin{aligned}
b_{0} & =\frac{A(\wp(p))}{\left(\wp(p)-\wp\left(a_{1}\right)\right)^{5}\left(\wp(p)-\wp\left(a_{2}\right)\right)^{2}\left(\wp(p)-\wp\left(a_{3}\right)\right)^{2}} \\
& \sim \frac{A(\wp(p))}{(3 \wp(p)-2 u)^{5}\left(9 \wp(p)^{2}+6 u \wp(p)+u^{2}-9\right)^{2}}
\end{aligned}
$$

where $A(\wp(p))$ is a polynomial of $\wp(p)$ of degree 9 .

The boundary behavior of close string moduli $u$ especially at the conifold point $u \rightarrow 1$ is discussed more details in section 6.4 .

\subsection{Holomorphic anomaly equation for open amplitudes}

We see that we can use the matrix model formalism to compute higher genus topological amplitude for the massless $N_{f}=2$ Seiberg-Witten theory. But the formalism gets quite complicated at higher genus, and for the close topological amplitude $F^{(g)}$, the most efficient

\footnotetext{
${ }^{20}$ They are too cumbersome to write down here, but are available upon request.

${ }^{21}$ The first remark follows generally from the Eynard formalism. The second remark applies to the $N_{f}<4$ $\mathrm{SU}(2)$ spectral curves and the cubic matrix model.
} 
way of calculation is still through the use the holomorphic anomaly equation plus boundary conditions at the conifold point. One might wonder whether this method of "direct integration" can also be applied to the open topological amplitude. In order to explore this idea, we consider a version of the holomorphic anomaly equation for the open topological amplitudes proposed in [49] based on the matrix model formalism.

An extended open holomophic anomaly equation has been applied to the calculations of open amplitudes on the the real quintic Calabi-Yau manifold [50]. This formalism was recently applied to local $\mathcal{O})(-3) \rightarrow \mathbb{P}^{2}[51]$. It differs from the discussion here, as it encorporates no open moduli.

The open holomorphic anomaly equation of [49] is

$$
\partial_{\bar{K}} W_{k}^{(g)}=\frac{1}{2} C_{\bar{K}}^{I J}\left(D_{I} D_{J} W_{k}^{(g-1)}+\sum_{h} \sum_{L \subset K} D_{I} W_{l}^{(h)} D_{J} W_{k-l}^{(g-h)}\right),
$$

where the $I, J, K$ are close string moduli. For our toy model of $N_{f}=2 \mathrm{SU}(2)$ SeibergWitten theory, the only anti-holomorphic dependence comes from the function $\hat{E}_{2}(\tau)$, which appears in the variable $X$ we defined in (6.13). The close string moduli in this case can be parametrized by the period $a$, and since it is a flat coordinate in the holomorphic limit, the covariant derivatives in the r.h.s. of (6.30) can be replaced by just ordinary derivatives. After fixing the normalization correctly, the equation (6.30) becomes for the case at hand

$$
-\frac{16}{\theta_{2}^{4}(\tau)} \frac{\partial W_{k}^{(g)}}{\partial X}=\partial_{a}^{2} W_{k}^{(g-1)}+\sum_{h} \sum_{L \subset K} \partial_{a} W_{l}^{(h)} \partial_{a} W_{k-l}^{(g-h)} .
$$

Consider the simplest case of the above open holomorphic anomaly equation, namely the case $g=0$ and $k=3$. The equation becomes

$$
\begin{aligned}
-\frac{16}{\theta_{2}^{4}(\tau)} \frac{\partial W_{3}^{(0)}(p, q, r)}{\partial X}= & 2 \partial_{a} W_{1}^{(0)}(p) \partial_{a} W_{2}^{(0)}(q, r)+2 \partial_{a} W_{1}^{(0)}(q) \partial_{a} W_{2}^{(0)}(p, r) \\
& +2 \partial_{a} W_{1}^{(0)}(r) \partial_{a} W_{2}^{(0)}(p, q)
\end{aligned}
$$

To test the equation, we can use the residue formulae to compute directly the genus zero 3-point function

$$
\begin{aligned}
W_{3}^{(0)}(p, q, r) & =\operatorname{Res}_{s \rightarrow \mathbf{a}} \frac{d E_{s}(p)}{\omega(s)}[B(s, q) B(\bar{s}, r)+B(\bar{s}, q) B(s, r)] \\
& =-\frac{\left(\wp\left(p-a_{1}\right)+X\right)\left(\wp\left(q-a_{1}\right)+X\right)\left(\wp\left(r-a_{1}\right)+X\right)}{\sqrt{2}\left(u^{2}-1\right)} .
\end{aligned}
$$

On the r.h.s., the genus zero one-point function is undefined in the matrix model formalism, and the notation of $\partial_{a} W_{1}^{(0)}(p)$ simply means the contour integral of $W_{2}^{(0)}(p, q)=B(p, q)$ over the B-cycle. We find

$$
\partial_{a} W_{1}^{(0)}(p)=\int_{0}^{2 a_{2}} B(p, q) d q=\frac{\pi i}{a_{1}}=\frac{2 \sqrt{2} i}{\theta_{2}^{2}(\tau)}
$$


We see an immediate problem with (6.32). The l.h.s. has a pole at $p \rightarrow a_{1}$, but the r.h.s. involves the Weierstrass Zeta function from $\partial_{a} \wp(p-q)$ and does not have a pole at $p \rightarrow a_{1}$. The discrepancy comes from the fact that in the derivation of the open holomorphic anomaly equation [49], the contour integral is converted into covariant derivative of the close string moduli. However, it seems that this procedure is not valid in the presence of open string moduli, so we have to do the contour integral directly instead of just taking derivative. Namely,

$$
\partial_{a} B(p, q) \neq \int_{0}^{2 a_{2}} W_{3}^{(0)}(q, r, s) d s
$$

So the correct version of the open holomorphic anomaly equation (6.32) should be

$$
-\frac{16}{\theta_{2}^{4}(\tau)} \frac{\partial W_{3}^{(0)}(p, q, r)}{\partial X}=2 \partial_{a} W_{1}^{(0)}(p) \int_{0}^{2 a_{2}} W_{3}^{(0)}(q, r, s) d s+\text { permutation. }
$$

We check this is indeed satisfied by plugging in the expression for genus zero 3-point function (6.33). However, this is not much useful for the purpose of computing $W_{3}^{(0)}(p, q, r)$ as it appears in both r.h.s. and l.h.s. .

We also consider the case $g=1$ and $k=1$. Using (6.24) we get

$$
-\frac{16}{\theta_{2}^{4}(\tau)} \frac{\partial W_{1}^{(1)}(p)}{\partial X}=\frac{4 \sqrt{2}}{3 \theta_{2}^{4}(\tau)\left(u^{2}-1\right)}\left[-3 X+2 u-3\left(\wp\left(p-a_{1}\right)+X\right)\right]
$$

Again the naive equation

$$
-\frac{16}{\theta_{2}^{4}(\tau)} \frac{\partial W_{1}^{(1)}(p)}{\partial X}=\partial_{a}^{2} W_{1}^{(0)}(p)+2 \partial_{a} W_{1}^{(0)}(p) \partial_{a} F^{(1)}
$$

is not correct, as it can be seen that the r.h.s. is independent of the open string modulus $p$ while the l.h.s. is dependent on $p$. The correct equation is

$$
-\frac{16}{\theta_{2}^{4}(\tau)} \frac{\partial W_{1}^{(1)}(p)}{\partial X}=-\int_{0}^{2 a_{2}} \int_{0}^{2 a_{2}} W_{3}^{(0)}(p, q, r) d q d r+2 \partial_{a} W_{1}^{(0)}(p) \partial_{a} F^{(1)}
$$

where the minus sign in the first term of r.h.s. is just due to the different conventions of using modular forms around conifold or infinity, and in the second term the derivative $\partial_{a} F^{(1)}$ is equal to the contour integral of genus one-point function since there is no open string moduli.

We summarize the findings in a few remarks.

1. The holomorphic anomaly equation (6.30) is oversimplified and the improved version does not seem to be too useful in computing higher point function $W_{k}^{(g)}$ when $k \geq 2$, because $W_{k}^{(g)}$ appears in both sides of the equation as exemplified by (6.36).

2. The reason for this subtlety in (6.35) is because we are using a non-standard differential one-form (6.17) necessary for our calculations in SU(2) Seiberg-Witten theory. If we used the standard differential one-form $\lambda=y d x$ as the [20], the open holomorphic anomaly equation would be valid, but this would not be the right differential one-form to compute the gravitational coupling of Seiberg-Witten theory. 
3. For the free energy $F^{(g)}$ and one-point function $W_{1}^{(g)}(p)$, the holomorphic anomaly equation can be used to determine the amplitudes up to a holomorphic anomaly. Only lower genus open amplitudes appear in the r.h.s. of the holomorphic anomaly equation. For example, in order to compute the genus two one-point amplitude $W_{1}^{(2)}(p)$ this way, we first have to determine lower amplitudes up to $F^{2}, W_{3}^{(1)}\left(p_{1}, p_{2}, p_{3}\right)$.

\subsection{Boundary condition for open topological amplitudes}

We now turn to another important issue of boundary conditions. We consider the limiting behavior open topological amplitudes around the conifold point, which is the point where $u \rightarrow 1, \tau_{D}=-\frac{1}{\tau} \rightarrow i \infty$, and

$$
a_{D}=-\frac{i}{3 \theta_{4}^{2}\left(\tau_{D}\right)}\left(E_{2}\left(\tau_{D}\right)-\theta_{3}^{4}\left(\tau_{D}\right)-\theta_{2}^{4}\left(\tau_{D}\right)\right) \rightarrow 0
$$

We now expand genus one one-point function (6.24) around the conifold point in terms of the flat coordinate $a_{D}$. Firstly it is convenient to rewrite the expression in terms of only $\wp=\wp(p), u$, and $X$

$$
\begin{aligned}
W_{1}^{(1)}(p)= & \frac{1}{2 \sqrt{2}\left(u^{2}-1\right)}\left\{X^{2}+\frac{3-3 u^{2}}{2 u-3 \wp} X+\frac{1}{36(2 u-3 \wp)^{2}\left(9 \wp^{2}+6 u \wp+u^{2}-9\right)} \times\right. \\
& {\left[405 u^{2}-1701\right) \wp^{4}+\left(216 u^{3}+648 u\right) \wp^{3}+\left(594 u^{4}+1620 u^{2}-486\right) \wp^{2} } \\
& \left.\left.+\left(384 u^{5}-4896 u^{3}+2592 u\right) \wp+65 u^{6}+501 u^{4}+675 u^{2}-729\right]\right\} .
\end{aligned}
$$

We notice the Weierstrass function $\wp(p)$ is also dependent on the underlying elliptic curve. However, for generic value of the open string modulus $p$, the function $\wp(p)$ has a finite generic value at the conifold point of the close string moduli space. So we can first expand $u$ and $X$ in the expression (6.41), and treat $\wp(p)$ as an independent parameter. Naively, we should expect the singular behavior as

$$
W_{1}^{(1)}(p)=\mathcal{O}\left(\frac{1}{a_{D}}\right)
$$

Surprisingly, we find that the leading singular term vanishes, and the conifold expansion is regular. The series expansion result is

$$
W_{1}^{(1)}(p)=\frac{3 \wp(p)-2}{8 \sqrt{2}(3 \wp(p)+4)}+\frac{i\left(9 \wp(p)^{2}+24 \wp(p)-8\right)}{8 \sqrt{2}(3 \wp(p)+4)^{2}} a_{D}+\mathcal{O}\left(a_{D}^{2}\right) .
$$

Thus the regularity of the conifold expansion in this case imposes boundary conditions for the open holomorphic ambiguity. For the genus one one-point function (6.41), the terms in the first line are fixed by the open holomorphic anomaly equation (6.39), and the rest is the ambiguity which can be parametrized by 14 constants in this case. Unfortunately, for generic holomorphic ambiguity, the coefficient of the singular $\frac{1}{a_{D}}$ term in the conifold expansion of $W_{1}^{(1)}(p)$ turns out to be a rational function of $\wp(p)$ whose numerator is a degree 4 polynomial of $\wp(p)$. So the conifold boundary condition only fixes 5 of the 14 unknown 
constants in the holomorphic ambiguity of $W_{1}^{(1)}(p)$. More ingenuity may be needed to completely fixes the holomorphic ambiguity.

We also similarly test the conifold expansion of the genus two one-point amplitude $W_{1}^{(2)}(p)$ in (6.28). The leading singular term with generic holomorphic ambiguity is $\mathcal{O}\left(\frac{1}{a_{D}^{3}}\right)$, but we again find that the actual series is not singular

$$
W_{1}^{(2)}(p)=\frac{27(3 \wp-2)}{512 \sqrt{2}(3 \wp+4)^{2}}+\frac{9 i\left(27 \wp^{3}+216 \wp^{2}+288 \wp-224\right)}{1024 \sqrt{2}(3 \wp(p)+4)^{3}} a_{D}+\mathcal{O}\left(a_{D}^{2}\right) .
$$

\section{$7 \quad$ Future directions}

We have solved the topological sector of the $N=2 \mathrm{SU}(2)$ gauge theories with $N_{f}=0,1,2,3$ matter multiplets in the fundamental representation. Near the asymptotic free region in the vector multiplet space our results agree with the instanton calculation of Nekrasov. At the conifold points and the conformal points our globally defined expressions predict the topological sector of these theories in canonical holomorphic coordinates.

Especially the analysis at the conformal points relies on the method proposed in [1]. It would be challenging and interesting to find a microscopic description especially at these points, at which the theory does not allow for an action formulation. The structure of the $F^{(g)}$ is very similar as at orbifold singularities in topological string theory $[19,37,46]$, which suggests that a dual string description is a serious candidate.

We described the construction of the modular objects entirely from the Picard-Fuchs system in a form that generalizes straightforwardly to $N=2$ theories with higher rank gauge groups and does not require knowledge of modular forms w.r.t. subgroups of $S P(2 g, \mathbb{Z})$. e.g. the solutions for the periods of [38] for $\mathrm{SU}(3)$ could be used to study the topological theory at Argyres-Douglas conformal points in $\mathrm{SU}(3)$ theory.

We find additional evidence that the simple boundary conditions namely the gap at the conifold and regularity of the amplitudes at the conformal points fix the entire ambiguity of $N=2$ topological theories associated to Riemann surfaces. However one should prove integrability of these type of topological theories in general.

Note that for the massless $N_{f}=4$ case the $F^{(g)}$ can be written as quasi-modular forms of weight $2 g-2$ of $\operatorname{PSL}(2, \mathbb{Z})[26]$ similar as the $F^{(g)}$ for the asymptotic free cases here, but there is no gap structure in the conformal cases. It seems possible but tedious to fix the ambiguity here by considering mass perturbations and the associate limits to the cases that are treated in this paper.

In the global case the above mentioned boundary conditions are not sufficient. We hope that this can be overcome by the study of various limit in multi-moduli compact CalabiYau manifold. For this reason we described the limit of rigid special Kähler geometry in great detail. Enough field theory limits, which are integrable, could make the global theory eventually also solvable.

We also compared our calculation with the matrix model, respectively spectral curve approach of Eynard and Orantin. This yields an alternative way to solve these theories, which gives additional information about certain open matrix model amplitudes, whose meaning has not been studied in the context of gauge theory yet. 
In [16] a microscopic matrix model for the Seiberg-Witten theory was derived starting from the instanton sums in asymptotic free regions. Here we go the opposite way and derive from the improved recursive formalism of [20] the global higher amplitudes, whose expansion in the asymptotic region checks with [4]. Given the by now well established relation of Seiberg-Witten gauge theory with the matrix model makes the gauge theory a most interesting laboratory to test the physical implications of the non-perturbative ideas that were recently put forward in the matrix model context $[52,53]$.

\section{Acknowledgments}

We thank Thomas Grimm, Babak Haghighat, Marcos Marino, Nicolas Orantin and Marco Rauch for fruitful discussions.

\section{A Nekrasov's calculations}

In [4] Nekrasov compute the Seiberg-Witten prepotential and its gravitational corrections by instanton counting. The results are represented by partition of instanton number into Young tableau. The results for $\mathrm{SU}(2)$ theory with one massive hypermultiplet, i.e. $N_{f}=1$, up to 5-instanton and genus $2 \operatorname{are}^{22}$

$$
\begin{aligned}
F^{(0)}= & 4 a^{2} \log (a)+\left(c_{2}^{(0)} a^{2}+c_{1}^{(0)} a+c_{0}^{(0)}\right) \\
& -\frac{1}{2}\left(a+m_{1}\right)^{2} \log \left(a+m_{1}\right)-\frac{1}{2}\left(-a+m_{1}\right)^{2} \log \left(-a+m_{1}\right) \\
& -\frac{m_{1}}{2 a^{2}}+\frac{3 a^{2}-5 m_{1}^{2}}{64 a^{6}}+\frac{7 a^{2} m_{1}-9 m_{1}^{3}}{192 a^{10}}-\frac{153 a^{4}-1430 a^{2} m_{1}^{2}+1469 m_{1}^{4}}{32768 a^{14}} \\
& -\frac{1131 a^{4} m_{1}-5250 a^{2} m_{1}^{3}+4471 m_{1}^{5}}{81920 a^{18}} \\
F^{(1)}= & \frac{1}{12} \log \left(\frac{(2 a)^{2}}{a^{2}-m_{1}^{2}}\right)+c^{(1)}-\frac{3 a^{2}-4 m_{1}^{2}}{128 a^{8}}-\frac{27 a^{2} m_{1}-32 m_{1}^{3}}{384 a^{12}} \\
& +\frac{9\left(73 a^{4}-733 a^{2} m_{1}^{2}+732 m_{1}^{4}\right)}{32768 a^{16}}+\frac{1899 a^{4} m_{1}-9259 a^{2} m_{1}^{3}+7848 m_{1}^{5}}{16384 a^{20}} \\
F^{(2)}= & -\frac{1}{480 a^{2}}+\frac{1}{240\left(a+m_{1}\right)^{2}}+\frac{1}{240\left(-a+m_{1}\right)^{2}} \\
& +\frac{9 a^{2}-11 m_{1}^{2}}{1024 a^{10}}+\frac{103 a^{2} m_{1}-117 m_{1}^{3}}{1024 a^{14}}-\frac{3\left(5583 a^{4}-58186 a^{2} m_{1}^{2}+57067 m_{1}^{4}\right)}{262144 a^{18}} \\
& -\frac{451719 a^{4} m_{1}-2273690 a^{2} m_{1}^{3}+1919923 m_{1}^{5}}{655360 a^{22}}
\end{aligned}
$$

Here $m_{1}$ is the mass of the hypermultiplet and for convenience we have set the $\mathcal{N}=$ 2 dynamical scale $\Lambda=1$, which can be easily recovered by dimensional analysis. The constants $c_{i}^{(g)}$ are not important for us. In the above formulae we have also included the leading perturbative terms. In $\mathrm{SU}(2)$ case the leading perturbative term at genus $g$ is $[4,5]$

$$
F_{\text {pert }}^{(g)}=\gamma_{g}(2 a)+\gamma_{g}(-2 a)-\sum_{i=1}^{N_{f}} \gamma\left(a+m_{i}\right)-\sum_{i=1}^{N_{f}} \gamma\left(-a+m_{i}\right)
$$

\footnotetext{
${ }^{22}$ Our convention has a sign difference from that of $[4,5]$ at odd genus.
} 
where

$$
\begin{aligned}
\gamma_{0}(x) & =\frac{1}{2} x^{2} \log (x)-\frac{3}{4} x^{2} \\
\gamma_{1}(x) & =\frac{1}{12} \log (x) \\
\gamma_{2}(x) & =-\frac{1}{240} \frac{1}{x^{2}} \\
\ldots & \\
\gamma_{g}(x) & =\frac{(-1)^{g} B_{2 g}}{2 g(2 g-2)} \frac{1}{x^{2 g-2}}, \quad g>1
\end{aligned}
$$

We will also consider the much simpler case where the hypermultiplets are massless. We list the results for $N_{f}=1,2,3$ and up to 5-instanton, genus 3. For $N_{f}=1$

$$
\begin{aligned}
& F^{(0)}=3 a^{2} \log (a)+\left(c_{2}^{(0)} a^{2}+c_{1}^{(0)} a+c_{0}^{(0)}\right)+\frac{3}{64 a^{4}}-\frac{153}{32768 a^{10}}+\cdots \\
& F^{(1)}=c^{(1)}-\frac{3}{128 a^{6}}+\frac{657}{32768 a^{12}}+\cdots \\
& F^{(2)}=\frac{1}{160 a^{2}}+\frac{9}{1024 a^{8}}-\frac{16749}{262144 a^{14}}+\cdots \\
& F^{(3)}=\frac{5}{2688 a^{4}}-\frac{3}{1024 a^{10}}+\frac{96453}{524288 a^{16}}+\cdots
\end{aligned}
$$

For $N_{f}=2$ we have

$$
\begin{aligned}
& F^{(0)}=2 a^{2} \log (a)+\left(c_{2}^{(0)} a^{2}+c_{1}^{(0)} a+c_{0}^{(0)}\right)-\frac{1}{2}-\frac{1}{64 a^{2}}-\frac{5}{32768 a^{6}}+\cdots \\
& F^{(1)}=-\frac{1}{6} \log (a)+c^{(1)}+\frac{1}{64 a^{4}}+\frac{23}{16384 a^{8}}+\cdots \\
& F^{(2)}=\frac{7}{480 a^{2}}-\frac{7}{1024 a^{6}}-\frac{1425}{262144 a^{10}}+\cdots \\
& F^{(3)}=\frac{31}{8064 a^{4}}+\frac{5}{2048 a^{8}}+\frac{8843}{524288 a^{12}}+\cdots
\end{aligned}
$$

For $N_{f}=3$ we have

$$
\begin{aligned}
& F^{(0)}=a^{2} \log (a)+\left(c_{2}^{(0)} a^{2}+c_{1}^{(0)} a+c_{0}^{(0)}\right)-\frac{1}{64}-\frac{1}{32768 a^{2}}+\cdots \\
& F^{(1)}=-\frac{1}{3} \log (a)+c^{(1)}-\frac{1}{128 a^{2}}+\frac{3}{32768 a^{4}}+\cdots \\
& F^{(2)}=\frac{11}{480 a^{2}}+\frac{5}{1024 a^{4}}-\frac{109}{262144 a^{6}}+\cdots \\
& F^{(3)}=\frac{47}{8064 a^{4}}-\frac{1}{512 a^{6}}+\frac{769}{524288 a^{8}}+\cdots
\end{aligned}
$$

Open Access. This article is distributed under the terms of the Creative Commons Attribution Noncommercial License which permits any noncommercial use, distribution, and reproduction in any medium, provided the original author(s) and source are credited. 


\section{References}

[1] M.-x. Huang and A. Klemm, Holomorphic anomaly in gauge theories and matrix models, JHEP 09 (2007) 054 [hep-th/0605195] [SPIRES].

[2] E. Witten, Quantum background independence in string theory, hep-th/9306122 [SPIRES].

[3] M. Aganagic, V. Bouchard and A. Klemm, Topological strings and (almost) modular forms, Commun. Math. Phys. 277 (2008) 771 [hep-th/0607100] [SPIRES].

[4] N.A. Nekrasov, Seiberg-Witten prepotential from instanton counting, Adv. Theor. Math. Phys. 7 (2004) 831 [hep-th/0206161] [SPIRES].

[5] N. Nekrasov and A. Okounkov, Seiberg-Witten theory and random partitions, hep-th/0306238 [SPIRES].

[6] S.H. Katz, A. Klemm and C. Vafa, Geometric engineering of quantum field theories, Nucl. Phys. B 497 (1997) 173 [hep-th/9609239] [SPIRES].

[7] N. Seiberg and E. Witten, Electric - magnetic duality, monopole condensation, and confinement in $N=2$ supersymmetric Yang-Mills theory, Nucl. Phys. B 426 (1994) 19 [Erratum ibid. B 430 (1994) 485] [hep-th/9407087] [SPIRES].

[8] N. Seiberg and E. Witten, Monopoles, duality and chiral symmetry breaking in $N=2$ supersymmetric QCD, Nucl. Phys. B 431 (1994) 484 [hep-th/9408099] [SPIRES].

[9] S. Kachru, A. Klemm, W. Lerche, P. Mayr and C. Vafa, Nonperturbative results on the point particle limit of $N=2$ heterotic string compactifications, Nucl. Phys. B 459 (1996) 537 [hep-th/9508155] [SPIRES].

[10] A. Klemm, W. Lerche, P. Mayr, C. Vafa and N.P. Warner, Self-dual strings and $N=2$ supersymmetric field theory, Nucl. Phys. B 477 (1996) 746 [hep-th/9604034] [SPIRES].

[11] A. Klemm, M. Mariño and S. Theisen, Gravitational corrections in supersymmetric gauge theory and matrix models, JHEP 03 (2003) 051 [hep-th/0211216] [SPIRES].

[12] R. Dijkgraaf and C. Vafa, Matrix models, topological strings and supersymmetric gauge theories, Nucl. Phys. B 644 (2002) 3 [hep-th/0206255] [SPIRES].

[13] R. Dijkgraaf and C. Vafa, On geometry and matrix models, Nucl. Phys. B 644 (2002) 21 [hep-th/0207106] [SPIRES].

[14] R. Dijkgraaf, A. Sinkovics and M. Temurhan, Matrix models and gravitational corrections, Adv. Theor. Math. Phys. 7 (2004) 1155 [hep-th/0211241] [SPIRES].

[15] B. Eynard, All orders asymptotic expansion of large partitions, J. Stat. Mech. (2008) P07023 [arXiv:0804.0381] [SPIRES].

[16] A. Klemm and P. Sulkowski, Seiberg-Witten theory and matrix models, Nucl. Phys. B 819 (2009) 400 [arXiv:0810.4944] [SPIRES].

[17] K. Hori and C. Vafa, Mirror symmetry, hep-th/0002222 [SPIRES].

[18] M. Mariño, Open string amplitudes and large order behavior in topological string theory, JHEP 03 (2008) 060 [hep-th/0612127] [SPIRES].

[19] B. Haghighat, A. Klemm and M. Rauch, Integrability of the holomorphic anomaly equations, JHEP 10 (2008) 097 [arXiv:0809.1674] [SPIRES].

[20] B. Eynard and N. Orantin, Invariants of algebraic curves and topological expansion, math-ph/0702045 [SPIRES]. 
[21] M. Bershadsky, S. Cecotti, H. Ooguri and C. Vafa, Kodaira-Spencer theory of gravity and exact results for quantum string amplitudes, Commun. Math. Phys. 165 (1994) 311 [hep-th/9309140] [SPIRES].

[22] P.C. Argyres, M. Ronen Plesser, N. Seiberg and E. Witten, New $N=2$ superconformal field theories in four dimensions, Nucl. Phys. B 461 (1996) 71 [hep-th/9511154] [SPIRES].

[23] V. Bouchard, A. Klemm, M. Mariño and S. Pasquetti, Remodeling the B-model, Commun. Math. Phys. 287 (2009) 117 [arXiv:0709.1453] [SPIRES].

[24] V. Bouchard, A. Klemm, M. Mariño and S. Pasquetti, Topological open strings on orbifolds, Commun. Math. Phys. 296 (2010) 589 [arXiv:0807.0597] [SPIRES].

[25] S. Yamaguchi and S.-T. Yau, Topological string partition functions as polynomials, JHEP 07 (2004) 047 [hep-th/0406078] [SPIRES].

[26] T.W. Grimm, A. Klemm, M. Mariño and M. Weiss, Direct integration of the topological string, JHEP 08 (2007) 058 [hep-th/0702187] [SPIRES].

[27] L.F. Alday, D. Gaiotto and Y. Tachikawa, Liouville correlation functions from four-dimensional gauge theories, Lett. Math. Phys. 91 (2010) 167 [arXiv:0906.3219] [SPIRES].

[28] M. Alim and J.D. Lange, Polynomial structure of the (open) topological string partition function, JHEP 10 (2007) 045 [arXiv: 0708.2886] [SPIRES].

[29] S. Hosono, BCOV ring and holomorphic anomaly equation, arXiv:0810.4795.

[30] A. Klemm, W. Lerche, S. Yankielowicz and S. Theisen, Simple singularities and $N=2$ supersymmetric Yang-Mills theory, Phys. Lett. B 344 (1995) 169 [hep-th/9411048] [SPIRES].

[31] E. D'Hoker, I.M. Krichever and D.H. Phong, The effective prepotential of $N=2$ supersymmetric $\mathrm{SU}\left(N_{c}\right)$ gauge theories, Nucl. Phys. B 489 (1997) 179 [hep-th/9609041] [SPIRES].

[32] W. Nahm, On the Seiberg-Witten approach to electric-magnetic duality, hep-th/9608121 [SPIRES].

[33] K. Ito and S.-K. Yang, Prepotentials in $N=2 \mathrm{SU}(2)$ supersymmetric Yang-Mills theory with massless hypermultiplets, Phys. Lett. B 366 (1996) 165 [hep-th/9507144] [SPIRES].

[34] D. Zagier, Elliptic modular forms and their applications, in The 1-2-3 of Modular Forms, Springer, Berlin Germany (2008).

[35] C. Vafa, A stringy test of the fate of the conifold, Nucl. Phys. B 447 (1995) 252 [hep-th/9505023] [SPIRES].

[36] D. Ghoshal and C. Vafa, $C=1$ string as the topological theory of the conifold, Nucl. Phys. B 453 (1995) 121 [hep-th/9506122] [SPIRES].

[37] M.-x. Huang, A. Klemm and S. Quackenbush, Topological string theory on compact Calabi-Yau: modularity and boundary conditions, Lect. Notes Phys. 757 (2009) 45 [hep-th/0612125] [SPIRES].

[38] A. Klemm, W. Lerche and S. Theisen, Nonperturbative effective actions of $N=2$ supersymmetric gauge theories, Int. J. Mod. Phys. A 11 (1996) 1929 [hep-th/9505150] [SPIRES]. 
[39] Y. Ohta, Prepotential of $N=2 \mathrm{SU}(2)$ Yang-Mills gauge theory coupled with a massive matter multiplet, J. Math. Phys. 37 (1996) 6074 [hep-th/9604051] [SPIRES].

[40] Y. Ohta, Prepotentials of $N=2 \mathrm{SU}(2)$ Yang-Mills theories coupled with massive matter multiplets, J. Math. Phys. 38 (1997) 682 [hep-th/9604059] [SPIRES].

[41] R. Fricke, Die modularen Funktionen und ihre Anwendungen, Bd 1 Teubner Leibzig (1916).

[42] A. Brandhuber and S. Stieberger, Periods, coupling constants and modular functions in $N=2 \mathrm{SU}(2)$ SYM with massive matter, Int. J. Mod. Phys. A 13 (1998) 1329 [hep-th/9609130] [SPIRES].

[43] T. Eguchi and H. Kanno, Topological strings and Nekrasov's formulas, JHEP 12 (2003) 006 [hep-th/0310235] [SPIRES].

[44] P.C. Argyres and M.R. Douglas, New phenomena in SU(3) supersymmetric gauge theory, Nucl. Phys. B 448 (1995) 93 [hep-th/9505062] [SPIRES].

[45] A. Klemm, On the geometry behind $N=2$ supersymmetric effective actions in four dimensions, hep-th/9705131 [SPIRES].

[46] M. Alim, J.D. Lange and P. Mayr, Global properties of topological string amplitudes and orbifold invariants, JHEP 03 (2010) 113 [arXiv: 0809.4253] [SPIRES].

[47] A. Klemm and E. Zaslow, Local mirror symmetry at higher genus, hep-th/9906046 [SPIRES].

[48] M. Aganagic, A. Klemm, M. Mariño and C. Vafa, Matrix model as a mirror of Chern-Simons theory, JHEP 02 (2004) 010 [hep-th/0211098] [SPIRES].

[49] B. Eynard, M. Mariño and N. Orantin, Holomorphic anomaly and matrix models, JHEP 06 (2007) 058 [hep-th/0702110] [SPIRES].

[50] J. Walcher, Extended holomorphic anomaly and loop amplitudes in open topological string, Nucl. Phys. B 817 (2009) 167 [arXiv:0705.4098] [SPIRES].

[51] D. Krefl and J. Walcher, The real topological string on a local Calabi-Yau, arXiv:0902.0616 [SPIRES].

[52] M. Mariño, R. Schiappa and M. Weiss, Multi-instantons and multi-cuts, J. Math. Phys. 50 (2009) 052301 [arXiv:0809.2619] [SPIRES].

[53] B. Eynard and M. Mariño, A holomorphic and background independent partition function for matrix models and topological strings, arXiv:0810.4273 [SPIRES].

[54] N. Seiberg and E. Witten, Monopoles, duality and chiral symmetry breaking in $N=2$ supersymmetric QCD, Nucl. Phys. B 431 (1994) 484 [hep-th/9408099] [SPIRES]. 